\title{
Fair Resource Allocation for OFDMA Femtocell Networks with Macrocell Protection
}

\author{
Vu Nguyen Ha, Student Member, IEEE and Long Bao Le, Senior Member, IEEE
}

\begin{abstract}
We consider the joint subchannel allocation and power control problem for OFDMA femtocell networks in this paper. Specifically, we are interested in the fair resource sharing solution for users in each femtocell that maximizes the total minimum spectral efficiency of all femtocells subject to protection constraints for the prioritized macro users. Toward this end, we present the mathematical formulation for the uplink resource allocation problem and propose an optimal exhaustive search algorithm. Given the exponential complexity of the optimal algorithm, we develop a distributed and low-complexity algorithm to find an efficient solution for the problem. We prove that the proposed algorithm converges and analyze its complexity. Then, we extend the proposed algorithm in three different directions, namely downlink context; resource allocation with rate adaption for femto users; and consideration of a hybrid access strategy where some macro users are allowed to connect with nearby femto base stations to improve the performance of the femto tier. Finally, numerical results are presented to demonstrate the desirable performance of the proposed algorithms.
\end{abstract}

Index Terms-Femtocell networks, resource allocation, interference management, OFDMA, power control, subchannel assignment.

\section{INTRODUCTION}

Massive deployment of small cells such as femtocells provides an important solution to fundamentally improve the indoor throughput and coverage performance of wireless cellular networks [1]. 4G and beyond cellular networks are based on Orthogonal Frequency Division Multiple Access (OFDMA) that provides flexibility in radio resource management and robustness against adverse effects of multi-path fading [2]. Hence, design of an OFDMA femtocell tier so that it can coexist efficiently with the existing macrocell tier is an important research topic. One particular challenge in realizing this objective stems from the fact that femtocells are randomly deployed and they operate on the same frequency band with the macrocell tier, which can create strong crosstier interference to the macrocells. Therefore, to successfully deploy the femtocell network, it is required to resolve many technical challenges which range from resource allocation, synchronization [3] to protection of existing macrocells against cross-tier interference from femtocells [4].

Copyright (C)2013 IEEE. Personal use of this material is permitted. However, permission to use this material for any other purposes must be obtained from the IEEE by sending a request to pubs-permissions@ieee.org.

Manuscript received March 14, 2013; revised August 02, 2013; accepted September 25, 2013. The review of this paper was coordinated by Dr. Y. T. Su.

The authors are with the Institut National de la Recherche ScientifiqueÉnergie, Matériaux et Télécommunications, Université du Québec, Montréal, QC H3C 3P8, Canada (e-mail: hanguyen@emt.inrs.ca; long.le@emt.inrs.ca).
There have been some existing works studying the resource allocation for OFDMA-based femtocell networks [512]. In [5], spectrum sharing and access control strategies are proposed for femtocell networks by using the water-filling algorithm and game theory technique. The authors in [6] propose two methods to mitigate the uplink interference for OFDMA femtocell networks. In the first method, Femtocell User Equipments (FUEs) that produce strong interference to Macrocell User Equipments (MUEs) are only allowed to use dedicated subchannels while the remaining FUEs can utilize all subchannels assigned for the femto tier. In the second method, an auction-based algorithm is devised to optimize the channel assignment for both tiers to mitigate the co-tier interference. Interference avoidance and resource allocation issues for OFDMA femtocell networks are also studied in [7] where a greedy algorithm is developed to enable selforganization of femtocells. In [8], Cheung et al. study the network performance where the macrocells and femtocells utilize separate sets of subchannels or share the whole spectrum under both open and closed access strategies. The QoS-aware admission control design for OFDMA femtocells is conducted in [9]. All these existing works, however, do not consider power control in their proposed resource allocation algorithms.

In [10], the authors develop an adaptive femtocell interference management algorithm comprising three control loops that run continuously and separately at macro and femto base stations (MBS and FBS) to determine initial femto maximum power, target signal-to-interference-plus-noise (SINRs) for FUEs, and to control the transmission power, respectively. However, subchannel assignment is not studied and the maximum power constraint for each user is not considered in this work. Spectrum sharing and power allocation are investigated in $[11,12]$. In particular, [11] aims to enhance the energy efficiency and while the main design objective of [12] is to achieve user-level fairness for cognitive femtocells. However, the algorithms developed in [11] and [12] do not provide QoS guarantees for users of both network tiers.

In this paper, we study the joint subchannel and power allocation problem for femtocell networks considering fairness for FUEs in each femtocell, protection of MUEs, and maximum power constraints. To the best of our knowledge, none of the existing works on the OFDMA based femtocell network jointly consider all these design issues. In particular, we make the following contributions.

- We formulate the uplink fair subchannel and power allocation problem, study its optimal structure, and present an algorithm to find its optimal solution. To overcome the exponential complexity of the centralized optimal ex- 
haustive search algorithm, we then develop a distributed and low-complexity resource allocation algorithm. The proposed algorithm iteratively updates the subchannel assignments at each femtocell based on carefully designed assignment weights and transmission powers for the subchannels accordingly. We prove the convergence of the proposed algorithm and analyze its complexity.

- We extend the proposed solution in three important directions. Specifically, we show how the proposed resource allocation algorithm can be adapted for the downlink context. Moreover, we develop a distributed algorithm for the scenario where FUEs in different femtocells are allowed to choose different target rates per subchannel to better adapt to the network interference. Finally, we extend the proposed algorithm to implement the hybrid spectrum access where some MUEs are permitted to connect to nearby FBSs.

- Numerical results are presented to demonstrate the efficacy of the proposed algorithms and their relative performance compared to the optimal algorithm. In particular, we demonstrate the impacts of target rates per subchannel (i.e., modulation schemes), maximum powers, rate adaptation, and hybrid access on the performance of the femtocell network.

The remaining of this paper is organized as follows. We describe the system model and the uplink problem formulation in Section II. In Section III, we present both optimal exhaustive search and sub-optimal resource allocation algorithms. Extensions for the downlink scenario, adaptive-rate transmission, and hybrid access control are described in Section IV. Numerical results are presented in Section $\mathrm{V}$ followed by conclusion in Section VI. Key notations used in the paper are summarized in Table III.

\section{System Model And Problem Formulation}

\section{A. System Model}

We consider the OFDMA-based two-tier macrocellfemtocell network where users of both tiers share the spectrum comprising $N$ subchannels. We assume that there are $M_{\mathrm{f}}$ FUEs served by $(K-1)$ FBSs, which are underlaid by one macrocell serving $M_{\mathrm{m}}$ MUEs. Let $\mathcal{U}_{k}$ be the set of user equipments (UEs) in the $k$-th cell, i.e., they are served by BS $k$ of the corresponding tier. For convenience, let $\mathcal{U}_{\mathrm{m}} \triangleq \mathcal{U}_{1}$ represent the set of MUEs and $\mathcal{U}_{\mathrm{f}} \triangleq \cup_{k=2}^{K} \mathcal{U}_{k}=\left\{M_{\mathrm{m}}+1, \ldots, M_{\mathrm{m}}+M_{\mathrm{f}}\right\}$ denote the set of all FUEs. In addition, let $\mathcal{U}$ and $\mathcal{B}$ be the sets of all UEs and BSs, respectively. Then, we have $\mathcal{U}=\mathcal{U}_{\mathrm{m}} \cup \mathcal{U}_{\mathrm{f}}=\{1,2, \ldots, M\}$ and $\mathcal{B}=\{1,2, \ldots, K\}$ where BS 1 is assumed to be the MBS and $\mathcal{B}_{\mathrm{f}}=\{2, \ldots, K\}$ denotes the set of all FBSs.

We assume the fixed BS association for all UEs of both tiers in the network (i.e., each UE is served by a fixed BS in the corresponding tier) to present the problem formulation in this section. Relaxation of this assumption under the hybrid access design will be considered in Section IV. Now, let $b_{i} \in \mathcal{B}$ denote the BS serving UE $i$ and let $\mathcal{N}=\{1,2, \ldots, N\}$ be the set of all orthogonal subchannels. We assume there is no interference among transmissions on different subchannels. We consider a system with full frequency reuse where all $N$ subchannels are allocated for UEs in all cells of either tier. To describe the subchannel assignments (SA), let $\mathbf{A} \in \mathfrak{R}^{M \times N}$ be the SA matrix for all $M$ UEs over $N$ subchannels where

$\mathbf{A}(i, n)=a_{i}^{n}= \begin{cases}1 & \text { if subchannel } n \text { is assigned for UE } i \\ 0 & \text { otherwise. }\end{cases}$

We assume that a subchannel can be allocated to at most one UE in any cell. Then, we have

$$
\sum_{i \in \mathcal{U}_{k}} a_{i}^{n} \leq 1, \quad \forall k \in \mathcal{B} \text { and } \forall n \in \mathcal{N} .
$$

These constraints will be applied to all resource allocation problems studied in this paper.

\section{B. Physical-layer Model}

We assume that MQAM modulation is adopted for communications on each subchannel where the constellation size $s$ for $s$-QAM is chosen from a predetermined set $\mathfrak{M}$. To guarantee acceptable performance when a constellation size $s$ is adopted on a particular subchannel, we need to ensure that the SINR on that subchannel is not smaller than a corresponding target SINR value $\bar{\gamma}(s)$. To determine $\bar{\gamma}(s)$, let $f(\gamma(s))$ be the expression of bit error rate (BER) for SINR $\gamma(s)$ when the constellation size $s$ is adopted. Suppose we want to maintain the BER to be not greater than a target value $\bar{P}_{e}$. Then, the SINR must satisfy the following for the constellation size $s$

$$
\gamma(s) \geq \bar{\gamma}(s)=f^{-1}\left(\bar{P}_{e}\right),
$$

where $f^{-1}($.$) denotes the inverse function of the BER$ function $f($.$) . To demonstrate this calculation, suppose that$ MQAM modulation with square signal constellations (i.e., $s$-QAM where $s \in \mathfrak{M} \triangleq\left\{4,16,64,256, \ldots, s_{\max }\right\}=\left\{2^{2 \sigma} \mid\right.$ $\sigma$ is a positive integer and $\left.\sigma \leq 1 / 2 \log _{2} s_{\max }\right\}$ ) is employed. According to [13] (equation (4.2.144)), the BER of the $s$-QAM scheme with Gray encoding can be approximated as

$$
f(\gamma(s)) \approx x_{s} \mathbb{Q}\left(\sqrt{y_{s} \gamma(s)}\right), s \in \mathfrak{M},
$$

where $\mathbb{Q}($.$) stands for the Q-function, x_{s}=\frac{2(1-1 / \sqrt{s})}{\log _{2} s}, y_{s}=$ $\frac{3}{2(s-1)}$ and $\gamma(s)$ is the SINR. Using the result in (3), the target SINR for the $s$-QAM modulation scheme can be calculated as

$$
\bar{\gamma}(s)=\frac{\left[\mathbb{Q}^{-1}\left(\bar{P}_{e} / x_{s}\right)\right]^{2}}{y_{s}}, s \in \mathfrak{M},
$$

where recall that $\bar{P}_{e}$ is the target BER value. We assume that ideal Nyquist data pulses are used where the symbol rate on each subchannel is equal to $B W / N$ where $B W$ is the total bandwidth of $N$ subchannels. Then, if $s$-QAM modulation scheme is employed, the spectral efficiency per one $\mathrm{Hz}$ of system bandwidth is $\frac{\log _{2} s}{N}(\mathrm{~b} / \mathrm{s} / \mathrm{Hz})$.

\section{Uplink Resource Allocation Problem}

Let $p_{i}^{n}$ represent the transmission power of UE $i$ over subchannel $n$ in the uplink where $p_{i}^{n} \geq 0$. We impose the following constraints on the total transmission powers

$$
\sum_{n=1}^{N} p_{i}^{n} \leq P_{i}^{\max }, \quad i \in \mathcal{U},
$$


where $P_{i}^{\max }$ is the maximum transmission power of UE $i$. Similar to the SAs, we define $\mathbf{P}$ as an $M \times N$ power allocation (PA) matrix where $\mathbf{P}(i, n)=p_{i}^{n}$. For convenience, we also define partitions of $\mathbf{S A}$ and PA matrices $\mathbf{A}$ and $\mathbf{P}$ as follows. In particular, let $\mathbf{A}_{k}, \mathbf{P}_{k} \in \mathfrak{R}^{\left|\mathcal{U}_{k}\right| \times N}$ represent the SA and PA matrices for UEs in cell $k$ over $N$ subchannels, respectively.

Let $h_{i j}^{n}$ and $\eta_{i}^{n}$ be the channel power gain from UE $j$ to BS $i$ and the noise power at BS $i$ over subchannel $n$, respectively. Then, for a given SA and PA solution, i.e., given $\mathbf{A}$ and $\mathbf{P}$, the SINR achieved at BS $b_{i}$ due to the transmission of UE $i$ over subchannel $n$ can be written as

$$
\Gamma_{i}^{n}(\mathbf{A}, \mathbf{P})=\frac{a_{i}^{n} h_{b_{i} i}^{n} p_{i}^{n}}{\sum_{j \notin \mathcal{U}_{b_{i}}} a_{j}^{n} h_{b_{i} j}^{n} p_{j}^{n}+\eta_{b_{i}}^{n}}=\frac{a_{i}^{n} p_{i}^{n}}{I_{i}^{n}(\mathbf{A}, \mathbf{P})},
$$

where $I_{i}^{n}(\mathbf{A}, \mathbf{P})$ is the effective interference corresponding to UE $i$ on subchannel $n$, which is defined as

$$
I_{i}^{n}(\mathbf{A}, \mathbf{P}) \triangleq \frac{\sum_{j \notin \mathcal{U}_{b_{i}}} a_{j}^{n} h_{b_{i} j}^{n} p_{j}^{n}+\eta_{b_{i}}^{n}}{h_{b_{i} i}^{n}} .
$$

We assume that SAs for MUEs have been determined by a certain mechanism and fixed while PA over the corresponding subchannels for MUEs are updated to cope with the cross-tier interference due to transmissions of FUEs. That means $\mathbf{A}_{1}$ is fixed while we need to determine $\mathbf{A}_{k}, 2 \leq k \leq K$ and the corresponding PAs. This assumption is quite natural since the macro tier has been deployed before the introduction of the femto tier; therefore, it would be more reasonable to keep the existing subchannel allocation mechanism and solution in the macro tier unchanged.

To protect the QoS of the licensed MUEs, we wish to maintain a predetermined target SINR $\bar{\gamma}_{i}^{n}$ for each of its assigned subchannel $n$. Specifically, we have the following constraints for the MUEs

$$
\Gamma_{i}^{n}(\mathbf{A}, \mathbf{P}) \geq \bar{\gamma}_{i}^{n}, \text { if } a_{i}^{n}=1, \quad \forall i \in \mathcal{U}_{\mathrm{m}},
$$

where $\bar{\gamma}_{i}^{n}=\bar{\gamma}\left(s^{\mathrm{m}}\right)$, which is calculated as in (3) if MUE $i$ employs $s^{\mathrm{m}}$-QAM modulation scheme.

For FUEs, we assume they all employ $s^{\mathrm{f}}$-QAM for a predetermined $s^{f}$. Then, the spectral efficiency (bits/s/Hz) achieved by FUE $i$ on one subchannel can be written as

$$
r_{i}^{n}(\mathbf{A}, \mathbf{P})= \begin{cases}0, & \text { if } \Gamma_{i}^{n}(\mathbf{A}, \mathbf{P})<\bar{\gamma}_{i}^{n}, \\ r_{\mathrm{f}}, & \text { if } \Gamma_{i}^{n}(\mathbf{A}, \mathbf{P}) \geq \bar{\gamma}_{i}^{n},\end{cases}
$$

where $r_{\mathrm{f}}=(1 / n) \log _{2} s^{\mathrm{f}}$ and $\bar{\gamma}_{i}^{n}=\bar{\gamma}\left(s^{\mathrm{f}}\right)$, which is calculated as in (3). Note that we have assumed that FUEs in all femtocells employ the same constellation size $s^{f}$ for ease of exposition. We will discuss the more general case in Section IV. In practice, we typically choose $s^{\mathrm{f}} \geq s^{\mathrm{m}}$ since FUEs can achieve higher transmission rates on each subchannel than MUEs thanks to their short distance to the associated FBS. This is indeed equivalent to $\bar{\gamma}\left(s^{\mathrm{f}}\right) \geq \bar{\gamma}\left(s^{\mathrm{m}}\right)$. In the analysis, we mostly use the same notation $\bar{\gamma}_{i}^{n}$ to refer to the required target SINR for FUEs or MUEs where $\bar{\gamma}_{i}^{n}=\bar{\gamma}\left(s^{\mathrm{f}}\right)$ for $\forall i \in \mathcal{U}_{\mathrm{f}}$ and $\bar{\gamma}_{i}^{n}=\bar{\gamma}\left(s^{\mathrm{m}}\right)$ for $\forall i \in \mathcal{U}_{\mathrm{m}}$. Now, we can express the total spectral efficiency achieved by UE $i$ for given SA and PA matrices $\mathbf{A}$ and $\mathbf{P}$ as

$$
R_{i}(\mathbf{A}, \mathbf{P})=\sum_{n=1}^{N} r_{i}^{n}(\mathbf{A}, \mathbf{P})
$$

To impose the max-min fairness for all FUEs associated with the same FBS, we define the minimum spectral efficiency of femtocell $k$ as the smallest value of spectral efficiencies of all FUEs in that femtocell, e.g.,

$$
\mathrm{R}^{(k)}(\mathbf{A}, \mathbf{P})=\min _{i \in \mathcal{U}_{k}} R_{i}(\mathbf{A}, \mathbf{P}) .
$$

The uplink resource allocation problem for FUEs can be formulated as follows:

$$
\begin{array}{r}
\max _{(\mathbf{A}, \mathbf{P})} \sum_{2 \leq k \leq K} \mathrm{R}^{(k)}(\mathbf{A}, \mathbf{P})=\sum_{2 \leq k \leq K} \min _{i \in \mathcal{U}_{k}} R_{i}(\mathbf{A}, \mathbf{P}) \\
\text { s. t. constraints } \quad(2),(6),(9),
\end{array}
$$

Therefore, the objective of this uplink resource allocation problem aims to balance between attaining an equal rate for FUEs in each femtocell (max-min fairness [25]) and high total femtocell spectral efficiency subject to SA constraints, FUE power constraints, and protection constraints for MUEs. Note that the SINR constraints for FUEs are embedded in the calculation of FUE spectral efficiency in (10). The resource allocation problem (13)-(14) can be transformed into a standard mixed integer program, which is, therefore, NP-hard.

\section{OPtimal AND SUP-OPtimal Algorithms}

In this section, we study the feasibility of a particular SA solution, which reveals the optimal structure of the optimization problem (13)-(14) based on which we develop optimal and suboptimal algorithms.

\section{A. Feasibility of a Subchannel Assignment Solution}

The resource allocation problem (13)-(14) involves finding a joint SA and PA solution. For a certain SA solution represented by matrix A satisfying the SA constraints (2), we can indeed find its "best" PA and verify its feasibility with respect to the constraints in (14). In particular, we wish to maintain the SINR constraints for FUEs and MUEs in (10) and (9), respectively. Specifically, for each subchannel $n$ we need to maintain SINRs constraints $\Gamma_{i}^{n}(\mathbf{A}, \mathbf{P}) \geq \bar{\gamma}_{i}^{n}$ for both MUEs and FUEs who are allocated with this subchannel.

Now, let $\mathcal{U}^{n}=\left\{i \in \mathcal{U} \mid a_{i}^{n} \neq 0\right\}=\left\{n_{1}, \ldots, n_{c^{n}}\right\}$ denote the set of UEs of both tiers who are assigned subchannel $n$ and $c^{n}=\left|\mathcal{U}^{n}\right|$ be the number of elements in set $\mathcal{U}^{n}$. Then, the SINR constraints for UEs in set $\mathcal{U}^{n}$, i.e., $\Gamma_{i}^{n}(\mathbf{A}, \mathbf{P}) \geq \bar{\gamma}_{i}^{n}, i \in$ $\mathcal{U}^{n}$, can be rewritten in matrix form as

$$
\left(\mathbf{I}^{n}-\mathbf{G}^{n} \mathbf{H}^{n}\right) \mathbf{p}^{n} \geq \mathbf{g}^{n},
$$

where $\mathbf{g}^{n}=\left[g_{n_{1}}^{n}, \ldots, g_{n_{c^{n}}}^{n}\right]^{T}$ with $g_{i}^{n}=\frac{\eta_{b_{i}}^{n} \bar{\gamma}_{i}^{n}}{h_{b_{i} i}^{n}}, \mathbf{I}^{n}$ is $c^{n} \times c^{n}$ identity matrix, $\mathbf{G}^{n}=\operatorname{diag}\left\{\bar{\gamma}_{n_{1}}^{n}, \ldots, \bar{\gamma}_{n_{c^{n}}}^{n}\right\}, \mathbf{p}^{n}=$ $\left[p_{n_{1}}^{n}, \ldots, p_{n_{c^{n}}}^{n}\right]^{T}$ and $\mathbf{H}^{n}$ is a $c^{n} \times c^{n}$ matrix defined as

$$
\left[\mathbf{H}_{i, j}^{n}\right]= \begin{cases}0, & \text { if } j=i \\ \frac{h_{b_{n_{i} n_{j}}}^{n}}{h_{b_{n_{i}} n_{i}}^{n}}, & \text { if } j \neq i .\end{cases}
$$

To study feasible solutions for inequality (15), we recall some standard results due to the Perron-Frobenius theorem [14], [22], [23] and their application to the power control problem [17]. Let $\lambda_{i}$ be the $i^{\text {th }}$ eigenvalue of matrix $\mathbf{G}^{n} \mathbf{H}^{n}$ 
and $\rho\left(\mathbf{G}^{n} \mathbf{H}^{n}\right)=\max _{i}\left|\lambda_{i}\right|$ be the maximum value of the modulus of all eigenvalues (i.e., the spectral radius of $\mathbf{G}^{n} \mathbf{H}^{n}$ ). The results in the Perron-Frobenius theorem can be applied for non-negative matrix $\mathbf{G}^{n} \mathbf{H}^{n}$, which is the case for our model since all power channel gains, noise powers, and target SINRs are real and non-negative numbers. Specifically, the PerronFrobenius theorem implies the following fact, which has been clearly stated in [17].

Fact: If $\mathbf{G}^{n} \mathbf{H}^{n}$ has non-negative elements, the following statements are equivalent:

1) There exists a non-negative power vector $\mathbf{p}$ such that $\left(\mathbf{I}^{n}-\mathbf{G}^{n} \mathbf{H}^{n}\right) \mathbf{p}^{n} \geq \mathbf{g}^{n}$.

2) $\rho\left(\mathbf{G}^{n} \mathbf{H}^{n}\right) \leq 1$.

3) $\left(\mathbf{I}-\mathbf{G}^{n} \mathbf{H}^{n}\right)^{-1}=\sum_{k=0}^{\infty}\left(\mathbf{G}^{n} \mathbf{H}^{n}\right)^{k}$ exists and is positive element-wise.

From these results, if $\rho\left(\mathbf{G}^{n} \mathbf{H}^{n}\right) \leq 1$ then we can find a solution of $\left(\mathbf{I}^{n}-\mathbf{G}^{n} \mathbf{H}^{n}\right) \mathbf{p}^{n}=\mathbf{g}^{n}$ (i.e., the equality case of the considered inequality) as

$$
\mathbf{p}^{n \star}=\left(\mathbf{I}-\mathbf{G}^{n} \mathbf{H}^{n}\right)^{-1} \mathbf{g}^{n},
$$

which is also the Pareto-optimal solution of $\left(\mathbf{I}^{n}\right.$ $\left.\mathbf{G}^{n} \mathbf{H}^{n}\right) \mathbf{p}^{n} \geq \mathbf{g}^{n}$ where Pareto-optimality means that any feasible solution $\mathbf{p}^{n}$ for the inequality $\left(\mathbf{I}^{n}-\mathbf{G}^{n} \mathbf{H}^{n}\right) \mathbf{p}^{n} \geq \mathbf{g}^{n}$ is not smaller than $\mathbf{p}^{n \star}$ element-wise (i.e., there not exist a feasible solution $\mathbf{p}^{n}$ for $\left(\mathbf{I}^{n}-\mathbf{G}^{n} \mathbf{H}^{n}\right) \mathbf{p}^{n} \geq \mathbf{g}^{n}$ so that $p_{i}^{n} \leq p_{i}^{n \star}, \forall i$ and $p_{j}^{n}<p_{j}^{n \star}$ for some $j$ ). These results has been used in [17] and several references therein.

In addition, this Pareto-optimal power vector can be achieved at the equilibrium by employing the following wellknown distributed Foschini-Miljanic power updates [16], [17]

$$
p_{i}^{n}(l+1):=p_{i}^{n}(l) \frac{\bar{\gamma}_{i}^{n}}{\Gamma_{i}^{n}(l)}=I_{i}^{n}(l) \bar{\gamma}_{i}^{n},
$$

where $\Gamma_{i}^{n}(l)$ and $I_{i}^{n}(l)$ are the SINR and effective interference achieved by $\mathrm{UE} i$ in iteration $l$, respectively. If there is no feasible solution for (15) (when $\rho\left(\mathbf{G}^{n} \mathbf{H}^{n}\right)>1$ ), the transmission powers due to (18) will increase to infinity [16], [17]. Now, we are ready to state one important result for a given SA solution A that satisfies the SA constraints (2) in the following proposition.

Proposition 1. Suppose that we can find a finite Paretooptimal PA solution $\mathbf{p}^{n}$ on the subchannel $n$ for the considered SA A, which is given in (17) (i.e., the spectrum radius $\left.\rho\left(\mathbf{G}^{n} \mathbf{H}^{n}\right)<1, \forall n\right)$. Then, the underlying $S A \mathbf{A}$ is feasible if these Pareto-optimal PA vectors $\mathbf{p}^{n}$ satisfy the power constraints in (6).

Proof. The Pareto-optimal PA solution $\mathbf{p}^{n}$ on each subchannel $n$ requires the minimum powers in the element-wise sense for all UEs, who are assigned subchannel $n$, to meet their SINR requirements. Therefore, the considered SA solution A is feasible if and only if the corresponding Pareto-optimal PA solutions $\mathbf{p}^{n}$ on all $N$ subchannels satisfy the power constraints in (6). Therefore, we have completed the proof of the proposition.

Since the number of possible SAs is finite, the result in this proposition pays the way for developing optimal exhaustive search algorithm, which is presented in the following.

\section{B. Optimal Algorithm}

1) Exhaustive Search Algorithm: Based on the results in section III-A, we can find the optimal solution for the resource allocation problem (13)-(14) by performing exhaustive search as follows. For a fixed and feasible $\mathbf{A}_{1},{ }^{1}$ let $\Omega\{\mathbf{A}\}$ be the list of all potential SA solutions that satisfy the SA constraints (2) and the fairness condition: $\sum_{n \in \mathcal{N}} a_{i}^{n}=\sum_{n \in \mathcal{N}} a_{j}^{n}=\tau_{k}$ for all FUEs $i, j \in \mathcal{U}_{k}$. Then, we sort the list $\Omega\{\mathbf{A}\}$ in the decreasing order of $\sum_{k=2}^{K} \tau_{k}$ to obtain the sorted list $\Omega^{*}\{\mathbf{A}\}$. Then, the feasibility of each SA solution in the list $\Omega^{*}\{\mathbf{A}\}$ can be verified as being presented in section III-A. Among all feasible SA solutions, the feasible one achieving the highest value of the objective function (13) and its corresponding PA solution given in (17) is the optimal solution of the optimization problem (13)-(14).

2) Complexity Analysis: The complexity of the exhaustive search algorithm can be determined by calculating the number of the elements in the list $\Omega^{*}\{\mathbf{A}\}$ and the complexity involved in the feasibility verification for each of them. The number of the elements in $\Omega^{*}\{\mathbf{A}\}$, the number of potential SAs, is the product of the number of potential SAs for all femtocells, which satisfy the "fairness condition". Therefore, the number of potential SAs can be calculated as

$$
\begin{aligned}
T & =\prod_{k=2}^{K} \sum_{\tau_{k}=0}^{\eta_{k}} \prod_{i=0}^{M_{k}-1} \mathrm{C}_{N-i \tau_{k}}^{\tau_{k}} \\
& =\prod_{k=2}^{K} \sum_{\tau_{k}=0}^{\eta_{k}} \frac{N !}{\left(\tau_{k} !\right)^{M_{k}}\left(N-M_{k} \tau_{k}\right) !} \approx O\left((N !)^{(K-1)}\right),
\end{aligned}
$$

where $\eta_{k}=\left\lfloor N / M_{k}\right\rfloor$ represents for the largest integer less than or equal to $N / M_{k}$ and $\mathrm{C}_{m}^{n}=\frac{m !}{n !(m-n) !}$ denotes the " $m$-choose- $n$ " operation. According to section III-A, the complexity of the feasibility verification for each potential SA mainly depends on the eigenvalue calculation of the corresponding matrix and solving the linear system to find the PA solution for each subchannel. It requires $O\left(K^{3}\right)$ to calculate the eigenvalues of $\mathbf{G}^{n} \mathbf{H}^{n}$ [18] and $O\left(K^{3}\right)$ to obtain $\mathbf{p}^{n}$ by solving a system of linear equations [19]. Therefore, the complexity of the optimal exhaustive search algorithm is $O\left(K^{3} \times N \times(N !)^{(K-1)}\right)$, which is exponential in the numbers of subchannels and FBSs. This optimal exhaustive search algorithm will serve as a benchmark to evaluate the low-complexity algorithm developed in the following.

\section{Sub-Optimal and Distributed Algorithm}

To resolve the exponential complexity of the centralized optimal algorithm presented in section III-B, we develop a low-complexity and distributed resource allocation algorithm. To achieve max-min fairness as required by the objective function (13), our algorithm aims to assign the maximum equal number of subchannels to FUEs in each femtocell and to perform Pareto-optimal PA for FUEs and MUEs on each subchannel so that they meet the SINR constraints in (10) and (9). To achieve this design goal, we propose a novel subchannel allocation weight metric so that high weights are

\footnotetext{
${ }^{1}$ The SA solution for MUEs corresponding to $\mathbf{A}_{1}$ is feasible if there exist a PA solution that satisfies the constraints in (9) when there is no femto tier
} 
assigned for "bad allocations" requiring large transmission powers and vice versa.

The proposed resource allocation algorithm is described in details in Algorithm 1. The key operation in this algorithm is the iterative weight-based SA that is performed in parallel at all femtocells. The SA weight for each subchannel and FUE pair is defined as the multiplication of the estimated transmission power and a scaling factor capturing the quality of the corresponding allocation. Specifically, each UE $i$ in cell $k$ estimates the transmission power on subchannel $n$ in each iteration $l$ of the algorithm by using the Foschini-Miljanic power update given in (18) as follows:

$$
p_{i}^{n, \min }=I_{i}^{n}(l) \bar{\gamma}_{i}^{n} .
$$

The effective interference $I_{i}^{n}(l)$ given in (8) can be estimated by UE $i$ as follows. The serving BS of UE $i$ estimates the power channel gain $h_{b_{i} i}^{n}$ on subchannel $n$ from this user to itself and transmits this channel state information to UE $i$. In addition, the BS of UE $i$ measures the total received power on subchannel $n$ due to both desired and interfering signals as well as the Gaussian noise, which is then sent back to UE $i$. UE $i$ can calculate $I_{i}^{n}(l)$ easily by using the gathered information (i.e., its transmission power, power channel gain to the BS, and the total received power at the BS) according to (8). We propose the following assignment weight for FUE $i$ on subchannel $n$ in cell $k$ as

$$
w_{i}^{n}=\chi_{i}^{n} p_{i}^{n, \min },
$$

where the scaling factor $\chi_{i}^{n}$ is defined as follows:

$$
\chi_{i}^{n}= \begin{cases}\alpha_{i}^{n}, & \text { if } p_{i}^{n, \text { min }} \leq \frac{P_{i}^{\max }}{\tau_{k}} \\ \alpha_{i}^{n} \theta_{i}^{n}, & \text { if } \frac{P_{i}^{\max }}{\tau_{k}}<p_{i}^{n, \min } \leq P_{i}^{\max } \\ \alpha_{i}^{n} \delta_{i}^{n}, & \text { if } P_{i}^{\max }<p_{i}^{n, \text { min }},\end{cases}
$$

where $\tau_{k}$ denotes the number of subchannels assigned for each FUE in femtocell $k ; \alpha_{i}^{n} \geq 1$ is a factor, which helps maintain SINR constraints of MUEs (i.e., it is increased if assigning subchannel $n$ to FUE $i$ tends to result in violation of the SINR constraint of the corresponding MUE); $\theta_{i}^{n}, \delta_{i}^{n} \geq 1$ are another factors which are set higher if the assignment of subchannel $n$ for FUE $i$ tends to require transmission power larger than the average power per subchannel (i.e., $\frac{P_{i}^{\text {max }}}{\tau_{k}}$ ) and the maximum power budget (i.e., $P_{i}^{\max }$ ), respectively. We will set $\delta_{i}^{n}$ as $\delta_{i}^{n}=$ $\mu_{i} \theta_{i}^{n}$ where $\mu_{i}=N$ in Algorithm 1. Given the weights defined for each FUE $i$, femtocell $k$ finds the SA for its FUEs by solving the following problem

$$
\begin{array}{cc} 
& \min _{\mathbf{A}_{k}} \sum_{i \in \mathcal{U}_{k}} \sum_{n \in \mathcal{N}} a_{i}^{n} w_{i}^{n} \\
\text { s.t. } \quad \sum_{n \in \mathcal{N}} a_{i}^{n}=\tau_{k}, \quad \forall i \in \mathcal{U}_{k} .
\end{array}
$$

This problem aims to find an assignment matrix $\mathbf{A}_{k}$ for which each FUE in femtocell $k$ is assigned $\tau_{k}$ subchannels with minimum total weight (i.e., to attain low-power SAs). The optimization problem (23) can be transformed into the standard matching problem (say between "jobs" and "employees") as follows. Suppose we create $\tau_{k}$ virtual "employees" for each FUE in femtocell $k$ then we can consider the matching problem between $\tau_{k} M_{k}$ virtual "employees" (virtual FUEs) and $N$ "jobs" (subchannels). In particular, FUE $i$ is equivalent to $\tau_{k}$ virtual FUEs $\left\{i_{1}, \ldots, i_{\tau_{k}}\right\}$. Let the edge $v_{i_{u}}^{n}\left(u \in\left\{1, \ldots, \tau_{k}\right\}\right)$ between subchannel $n$ and virtual FUE $i_{u}$ represent the assignment of that subchannel to the corresponding FUE. Then, the weight $w_{i_{u}}^{n}$ of the edge $v_{i_{u}}^{n}$ is equal to $w_{i}^{n}$. After performing this transformation, the SA solution of the problem (23) can be found by using the standard Hungarian algorithm (i.e., Algorithm 14.2.3 given in [20]). After running the Hungarian algorithm, if there exits a virtual FUE $i_{u}, u \in\left\{1, \ldots, \tau_{k}\right\}$, being matched with subchannel $n$, then we have $a_{i}^{n}=1$; otherwise, we set $a_{i}^{n}=0$. For further interpretation of Algorithm 1, let $W_{k}(l)$ denote the total minimum weight due to the optimal solution of (23). The main operations of Algorithm 1 can be summarized as follows.

- In steps 2-10, the MBS needs to estimate the effective interference on all subchannels and calculates the transmission powers for the corresponding MUEs by using (20). Then, the MBS checks the power constraints (6) for its associated MUEs. For MUEs that can maintain the power constraints (6), the MBS will send the newlycalculated transmission powers to them so that MUEs can update their powers accordingly (step 5). Otherwise, if the power constraint of any MUE is violated, the MBS will scale down the transmission powers to maintain the power constraints (step 7) and send the corresponding transmission powers to its MUEs. In addition, the MBS will inform all FBSs which will find the FUE creating the largest interference to the victim MUE on the subchannel with the largest transmission power; then we increase the parameter $\alpha_{m_{i}^{*}}^{n_{i}^{*}}$ corresponding to this FUE and subchannel pair by a factor of 2 (steps 8 and 9). This can be realized by allowing the MBS to request nearby FBSs to report the transmission power on the victim subchannel based on which the MBS can identify and request the most interfering FUE $m_{i}^{*}$ to update its parameter $\alpha_{m_{i}^{*}}^{n_{i}^{*}}$. The signaling required by these operations can be accommodated by the wired backhaul links (e.g., DSL links).

- In steps 15-20, each femtocell which has any FUEs's power constraints being violated in the previous iteration solves the SA problem (23) with the current weight values to update its SA solution. In addition, FBS $k$ decreases the target number of assigned subchannels $\tau_{k}$ by one if the optimal total weight $W_{k}(l)$ of the problem (23) satisfies the condition in step 17. Each FBS can complete all required tasks in these steps since it knows current transmission powers and scaling factors $\chi_{i}^{n}$ of its FUEs based on which it can calculate all SA weights.

- In step 12, each FBS estimates the effective interference on all subchannels and calculates the transmission powers for its FUEs by using (20). Then, each FBS will check the power constraints of its FUEs using the newly-calculated transmission powers. For FUEs that have their power constraints satisfied, the corresponding FBS will send the newly-calculated transmission powers to them so that they can update powers accordingly (steps 22 and 23). For any FUE that has its power constraint violated, its FBS 
scales down the transmission powers to meet the power constraints (step 25) and increase the $\theta_{i}^{n_{i}^{*}}$ parameter for the most interfering subchannel by a factor of 2 (steps 26 and 27). In both cases, each FBS must send the transmission power levels on all subchannels to the corresponding FUEs by using available control channels.

It can be observed that Algorithm 1 can be implemented distributively. In fact, the signaling information required in steps 8-9 must be sent over the wired backhaul link. In addition, each BS of either tier only needs to collaborate with its associated UEs to conduct all required tasks in other steps where the required signaling is sent over the air by using available control channels.

\section{Convergence and Complexity Analysis of Algorithm 1}

1) Convergence Analysis: The convergence of Algorithm 1 is stated in the following.

Proposition 2. Algorithm 1 converges to a feasible solution $(\mathbf{A}, \mathbf{P})$ of the optimization problem (13)-(14) .

Proof. To prove this proposition, we will consider two possible scenarios. For the first scenario, there exists an iteration after which the scaling factors $\chi_{i}^{n}$ for SA weights given in (22) do not change. According to Algorithm 1, after this iteration the SA solution will remain unchanged. In addition, Algorithm 1 simply updates transmission powers $p_{i}^{n, \mathrm{~min}}$ for all UEs on their assigned subchannels by using the Foschini-Miljanic power updates. Therefore, the authors in [16] have shown that these power updates indeed converge, which implies the convergence of Algorithm 1.

Now suppose that the scaling factors $\chi_{i}^{n}$ are still changed over iterations, we will prove that the system will ultimately evolve into the first scenario discussed above. First, it can be verified that the power $p_{i}^{n \text {,min }}$ given in (20) is lowerbounded by $\bar{\gamma}_{i}^{n} \eta_{b_{i}}^{n} / h_{b_{i} i}^{n}$. Therefore, if the scaling factors $\chi_{i}^{n}$ keep increasing over iterations then the total weight $W_{k}(l)$ returned by the assignment problem (23) will increase over iterations as well. Therefore, there exist some femtocells $k$ that decrease their number of assigned subchannels $\tau_{k}$ over iterations (steps 17-19 of Algorithm 1). Since initial values of all $\tau_{k}$ are finite, this process will terminate after a finite number of iterations. Then, the system will be in the first scenario discussed above; therefore, Algorithm 1 converges. Therefore, we have completed the proof of the proposition.

2) Complexity Analysis and Comparison with Existing Algorithms: It is observed that the major complexity of Algorithm 1 is involved in solving the SA by using the Hungarian method in step 16. According to Theorem 14.2.4. of [20], the complexity of the Hungarian algorithm is $O\left(N^{3}\right)$. Therefore, the complexity of our proposed algorithm is $O\left(K \times N^{3}\right)$ for each iteration. However, the local SAs can be performed in parallel at all $(K-1)$ femtocells. Therefore, the runtime complexity of our algorithm is $O\left(N^{3}\right)$ multiplied by the number of required iterations, which is quite moderate according to our simulation results (i.e., tens of iterations).

For comparison purposes, we summarize how existing resource allocation formulations and algorithms for two-tier

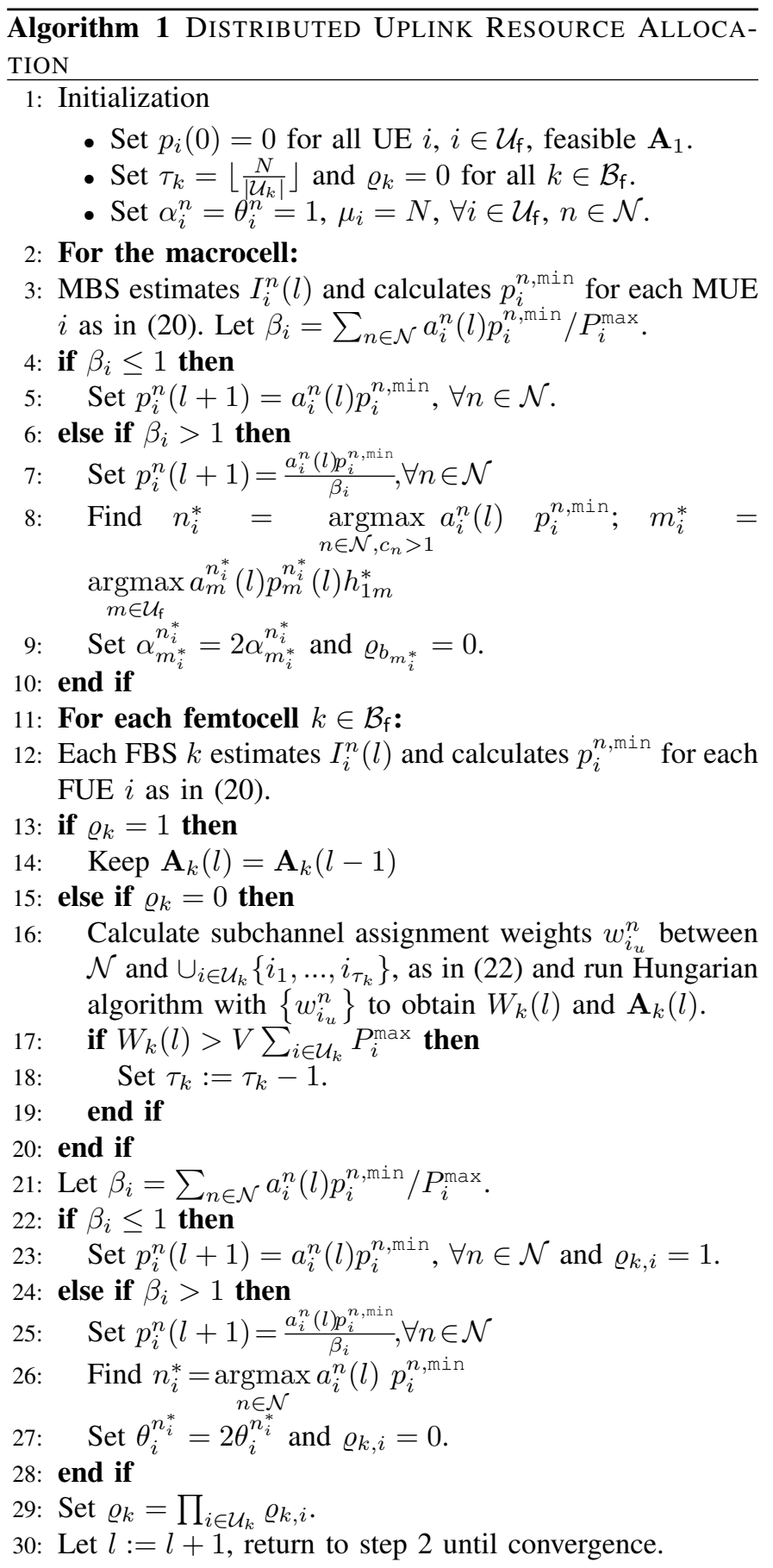

macro-femto networks cover different design aspects in Table I where we write NA for the convergence and complexity aspects if they are not analyzed in these existing works. As we can see, the existing works consider different optimization objectives and cover some design issues even though none of them accounts for all aspects. Our proposed algorithm captures all design aspects except the downlink scenario. Moreover, only Zhang et. al. [27] and we consider the power constraints in our resource allocation. However, Zhang et. al. aim to maximize the total throughput of the femtocell network while constraining the cross-tier interference from FUEs to the macro base station (BS). In their work, the co- 
tier interference among femtocells is assumed to be part of the noise power, which is not explicitly managed. Given the comparable design of Zhang's and our proposed algorithm, we will conduct performance comparison for the two algorithms in terms of throughput and fairness in Section V.

\section{FURTHER EXTENSIONS}

\section{A. Downlink Resource Allocation}

For the downlink context, we can use the same notations as in the uplink system. However, $h_{i j}^{n}$ and $\eta_{i}^{n}$ are the power channel gain from BS $j$ UE $i$ and noise power at $\mathrm{UE} i$, respectively. In the downlink system, we need to impose the power constraints for the BSs instead of UEs as follows:

$$
\sum_{i \in \mathcal{U}_{k}} \sum_{n \in \mathcal{N}} p_{i}^{n} \leq P_{\mathrm{BS}, k}^{\max }, \quad k \in \mathcal{B},
$$

where $P_{\mathrm{BS}, k}^{\max }$ is the maximum power of $\mathrm{BS} k$. Then, the downlink resource allocation problem can be formulated as

$$
\max _{(\mathbf{A}, \mathbf{P})} \sum_{2 \leq k \leq K} \mathrm{R}^{(k)}(\mathbf{A}, \mathbf{P})
$$

s. t. constraints (2), (9), (24).

We can employ Algorithm 1 to find a feasible solution of this problem with only some changes in the scaling factor $\chi_{i}^{n}$ of the assignment weight $w_{i}^{n}=\chi_{i}^{n} p_{i}^{n, \text { min }}$ as follows:

$$
\chi_{i}^{n}= \begin{cases}\alpha_{i}^{n}, & \text { if } p_{i}^{n, \min } \leq P_{\mathrm{BS}, b_{i}}^{\max } \\ \infty, & \text { if } p_{i}^{n, \min }>P_{\mathrm{BS}, b_{i}}^{\max } .\end{cases}
$$

In fact, the structure of power constraints in the downlink context is less complicated than those in the uplink setting since we only need to maintain the total power constraint for each BS instead of all UEs connected with each BS. This explains the simpler structure of (27) compared to that in (22).

The signaling requirements for the downlink case is a bit different from that in the uplink one, which is described in the following. To complete the tasks in steps 2-10, each MUE needs to estimate the current effective interference on their allocated subchannels based on which the MUE calculates the transmission powers on the corresponding subchannels. All MUEs then send the updated transmission powers to the MBS. The MBS updates the transmission powers on all subchannels accordingly if the power constraint (24) is satisfied (step 5). Otherwise, if the power constraint of the MBS is violated, the MBS scales down transmission powers on all subchannels by a factor $\beta^{1}=\sum_{n \in \mathcal{N}} \sum_{i \in \mathcal{U}_{1}} a_{i}^{n}(l) p_{i}^{n \text {,min }} / P_{\mathrm{BS}, 1}^{\max }$ to maintain the power constrailt. In addition, the MBS informs all FBSs which will find the FUE creating the largest interference to the victim MUE and increase the parameter $\alpha_{m_{i}^{*}}^{n_{i}^{*}}$ by a factor of 2 (steps 8 and 9). Here, the victim MUE $i$ is the one which is allocated subchannels $n_{i}^{*}$ satisfying $n_{i}^{*}=\operatorname{argmax}_{n \in \mathcal{N}, c_{n}>1} a_{i}^{n}(l)$ $p_{i}^{n, \min }$

The signaling in these tasks can be realized as follows. MUEs report the new transmission powers of their subchannels to the MBS via a control channel. Also, the MBS requests nearby FBSs to report the transmission powers on subchannel $n_{i}^{*}$ based on which the MBS can identify and request the most interfering FUE $m_{i}^{*}$ on subchannel $n_{i}^{*}$ to update its parameter $\alpha_{m_{i}^{*}}^{n_{i}^{*}}$ by using the wired backhaul links (e.g., DSL links). In steps 15-20, each FBS can complete all required tasks in these steps by requiring its FUEs to report newlycalculated transmission powers based on which it can calculate all SA weights. Note that each FBS knows the current values of scaling factor $\chi_{i}^{n}$ of the SA weights. The required signaling between FUEs and their corresponding FBSs can be accommodated by using available wireless control channels. Finally, each FBS that has its power constraint satisfied updates its transmission power (steps 22 and 23). Also, any FBS $k$ that has its power constraint violated scale downs transmission powers by a factor $\beta^{k}=\sum_{n \in \mathcal{N}} \sum_{i \in \mathcal{U}_{k}} a_{i}^{n}(l) p_{i}^{n, \min } / P_{\mathrm{BS}, k}^{\max }$ and increases the $\theta_{i}^{n_{i}^{*}}$ parameter for the subchannel with largest transmission power by a factor of 2 (steps 26 and 27).

\section{B. Adaptive-Rate Resource Allocation}

We can relax the fixed rate assumption and enable FUEs in each femtocell $k$ to choose any constellation size (modulation scheme) $s_{k} \in \mathfrak{M}$ for transmissions on their assigned subchannels. The target SINRs for different modulation schemes $s_{k}$-QAM $\bar{\gamma}\left(s_{k}\right)$ can be calculated as in (3). Hence, for a given $\left(s_{k}, \tau_{k}\right)$ the minimum spectral efficiency achieved by each FUE in femtocell $k$ is

$$
\mathrm{R}^{(k)}\left(s_{k}, \tau_{k}\right)=r_{k} \tau_{k},
$$

where $\tau_{k}$ is the number of subchannels assigned for each FUE in femtocell $k$ and $r_{k}=\frac{\log _{2} s_{k}}{N}$. The resource allocation problem for this adaptive-rate context is the same with (13)(14) except that different femtocells $k$ can choose different constellation size $s_{k} \in \mathfrak{M}$. Hence, we have following SINR constraints for each FUE $i$ connected with FBS $b_{i}$

$$
\Gamma_{i}^{n}(\mathbf{A}, \mathbf{P}) \geq \bar{\gamma}\left(s_{b_{i}}\right), \forall i \in \mathcal{U}_{\mathrm{f}}, s_{b_{i}} \in \mathfrak{M} .
$$

The equal number of subchannels assigned to each FUE in femtocell $k$ is at most $\left\lfloor N / M_{k}\right\rfloor$ and the maximum spectral efficiency transmitted over each subchannel is $\bar{r}=\log _{2} s_{\max } / N$ where $s_{\max }$ is the maximum allowable constellation size in $\mathfrak{M}$. Hence, the maximum spectral efficiency achieved by any FUE is $\bar{r}\left\lfloor N / M_{k}\right\rfloor$.

Resource allocation algorithm for this adaptive-rate setting can be performed by slightly adapting Algorithm 1, which is described in Algorithm 2. We list and sort all possible couples $\left(s_{k}, \tau_{k}\right)$ in the decreasing order of their $\mathrm{R}^{(k)}\left(s_{k}, \tau_{k}\right)=r_{k} \tau_{k}$ to obtain a sorted list $\Theta_{k}$. Then, the weight-based subchannel assignments can be performed by iteratively updating $\left(s_{k}, \tau_{k}\right)$ for all femtocells in the same order of the sorted list. In particular, if the current pair $\left(s_{k}, \tau_{k}\right)$ of any femtocell $k$ results in violation of the condition as specified in step 10 of Algorithm 2, then we update the pair $\left(s_{k}, \tau_{k}\right)$ by the next one in the list $\Theta_{k}$ (i.e., the element $\left(t_{k}+1\right)$ in the sorted list $\Theta_{k}$ is chosen), which has lower value of $\mathrm{R}^{(k)}\left(s_{k}, \tau_{k}\right)$. These updates are intuitive since the search space for this adaptive-rate setting comprises both the number of subchannels assigned for each FUE $\left(\tau_{k}\right)$ and the modulation scheme represented by $s_{k}$. These operations are repeated until convergence. 
TABLE I

SUMmary OF EXISTING ALGORITHMS

\begin{tabular}{|c|c|c|c|c|c|c|c|c|}
\hline Papers & Objective function & UL/DL & SA/PA & Prt. MUE & Pw. Const. & CoTI/CrTI & Conv. & Complexity \\
\hline Ref. [5] & Max-min number of assigned subchannels & Both & SA & Yes & No & No & NA & NA \\
\hline Ref. [6] & Max sum rate & UL & SA & Yes & No & Both & $\overline{\mathrm{NA}}$ & NA \\
\hline Ref. [7] & Max sum PRB & Both & SA & No & No & NA & NA & $O(K N \log N)$ \\
\hline Ref. [10] & $\begin{array}{l}\text { Min error between interference and prede- } \\
\text { termined thresholds at MUEs }\end{array}$ & $\overline{\mathrm{UL}}$ & Both & Yes & No & Both & NA & NA \\
\hline Ref. [11] & Max energy efficiency & Both & Both & No & No & No & Yes & NA \\
\hline Ref. [12] & Max-min effective rate & DL & PA & No & No & CoTI & NA & NA \\
\hline Zhang's paper & Max sum rate & $\mathrm{UL}$ & Both & Yes & Yes & CrTI & NA & NA \\
\hline Our paper & Max-min rate & UL & Both & Yes & Yes & Both & Yes & $O\left(K N^{3}\right)$ \\
\hline \multicolumn{4}{|c|}{$\begin{array}{l}\text { UL: Uplink } \\
\text { SA: Subchannel assignment } \\
\text { Prt. MUE: Protect the QoS of MUEs } \\
\text { CoTI: Co-tier interference among femtocells } \\
\text { Conv.: Convergence guarantee }\end{array}$} & \multicolumn{5}{|c|}{$\begin{array}{l}\text { DL: Downlink } \\
\text { PA: Power allocation } \\
\text { Pw. Const.: Power constraint } \\
\text { CrTI: Cross-tier interference between macrocell and femtocells } \\
\text { PRB: Physical resource block (frequency and time) }\end{array}$} \\
\hline
\end{tabular}

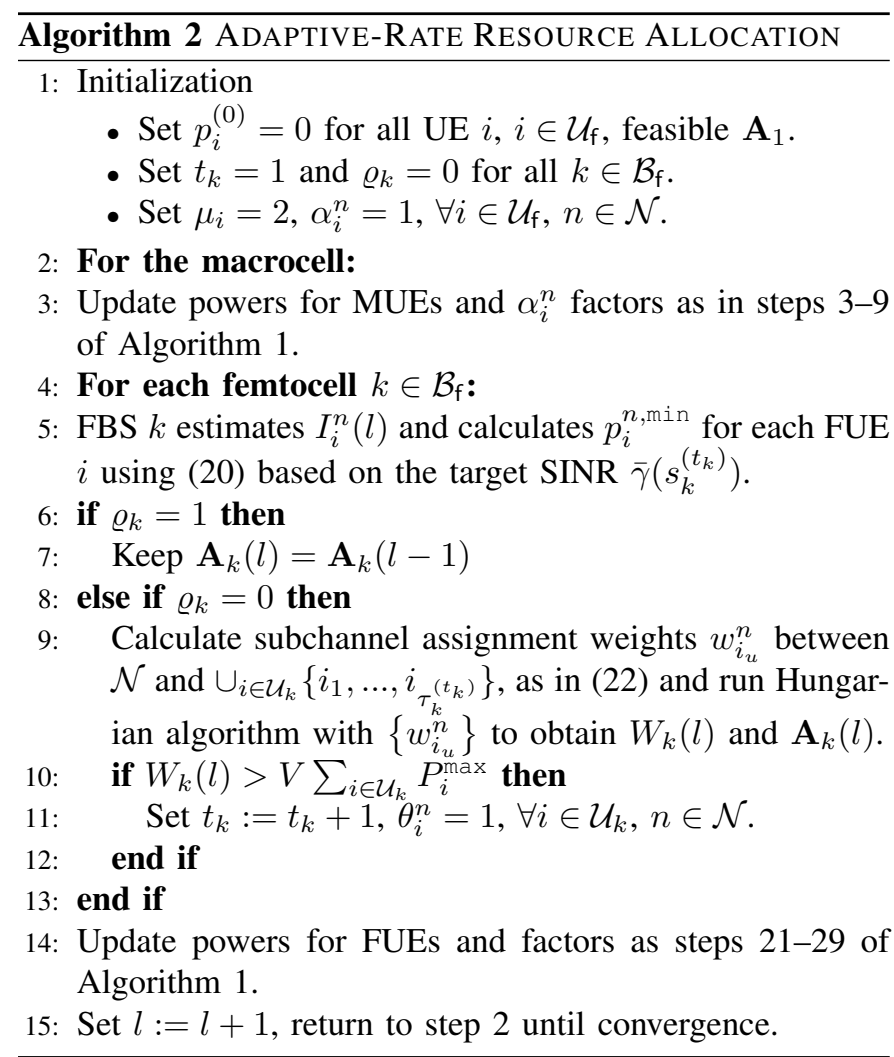

\section{Resource Allocation with Hybrid Access}

We can extend the considered problem to implement a hybrid access strategy, which can maintain the required QoS of MUEs while enhancing the performance of the femtocell tier. It can be observed that in the proposed algorithms (Algorithms $1,2)$, there may exist femtocells that do not utilize all available subchannels since doing so may prevent them from maintaining the target SINRs for all FUEs and MUEs. Therefore, it may be beneficial if we allow MUEs that potentially create and/or suffer from strong cross-tier interference from nearby femtocells to change their connections from the MBS to one nearby FBS. However, this can only be performed if the subchannels assigned to the underlying MUE are not utilized by the target femtocell.
We present the resource allocation algorithm with rate adaptation under such hybrid access in Algorithm 3. To interpret the operations of this algorithm, we first give definitions of involved quantities and parameters in the following. Let $b_{i}(l)$ and $\mathcal{N}_{i}(l)$ be the BS of UE $i$ and the set of its assigned subchannels in iteration $l$, respectively. Note that we have assumed that each MUE has a fixed set of assigned subchannels and FUEs have fixed associated FBSs. Therefore, we have $b_{i}(l)=b_{i}$ for each FUE $i$ and $\mathcal{N}_{i}(l)=\mathcal{N}_{i}$ for each MUE $i$ as specified by $\mathbf{A}_{1}$. In addition, we can estimate the total required transmission power over the assigned subchannels of MUE $i$ that is connected with BS $k$ as

$$
P_{i, k}=\bar{\gamma}\left(s^{\mathrm{m}}\right) \sum_{n \in \mathcal{N}_{i}} I_{i, k}^{n}(l)
$$

where $I_{i, k}^{n}(l)$ is the effective interference corresponding to MUE $i$ for its connection with BS $k$ on subchannel $n$, which can be calculated as in (8). MUE $i$ can determine $P_{i, k}$ for each potential FBS $k$ by estimating $I_{i, k}^{n}(l)$ on each assigned subchannel $n$. This can be realized as we have discussed in page 13 right below equation (20). For the SA in any iteration $l$, we define the set of potential FBSs for MUE $i$ as follows:

$$
\begin{aligned}
\mathcal{B}_{i}(l)= & \left\{k \mid k \in \mathcal{B}_{\mathrm{f}}, \mathcal{N}_{i} \subseteq \mathcal{N} / \cup_{j \in \mathcal{U}_{k}} \mathcal{N}_{j}(l-1),\right. \\
& \left.P_{i, k}<\min \left(P_{i, 1}, P_{i}^{\max }\right),\left|\mathcal{N}_{i}\right| \leq Q_{k}\right\}
\end{aligned}
$$

where $Q_{k}$ is the number of unused subchannels at FBS $k$ in the underlying iteration. Here, $\mathcal{B}_{i}(l)$ is the set of FBSs whose set of unused subchannels contains the set $\mathcal{N}_{i}$ of MUE $i$, connection between MUE $i$ with the underlying FBS requires less power than connection between MUE $i$ and the MBS. We also define the set $\mathcal{U}_{m, k}(l)=\left\{i \in \mathcal{U}_{\mathrm{m}} \mid b_{i}(l)=k\right\}$ which is the set of MUEs connecting with BS $k$ in iteration $l$.

In Algorithm 3, we do not change existing associations between any FBS $k$ and its corresponding MUEs until the chosen pair $\left(s_{k}, \tau_{k}\right)$ in this femtocell is updated (i.e., the next pair $\left(s_{k}, \tau_{k}\right)$ in the sorted list $\Theta_{k}$ is chosen). Specifically, if $t_{k}$ is updated (i.e., in step 13) then all MUEs currently connected with FBS $k$ are forced to change their connections back to the MBS (step 14); otherwise, we maintain the BS associations of all MUEs as they are (steps 15-16). In addition, only MUEs currently connecting with the MBS (i.e., any MUE $i$ with $b_{i}(l)$ equal 1) are allowed to change their BS association in iteration 


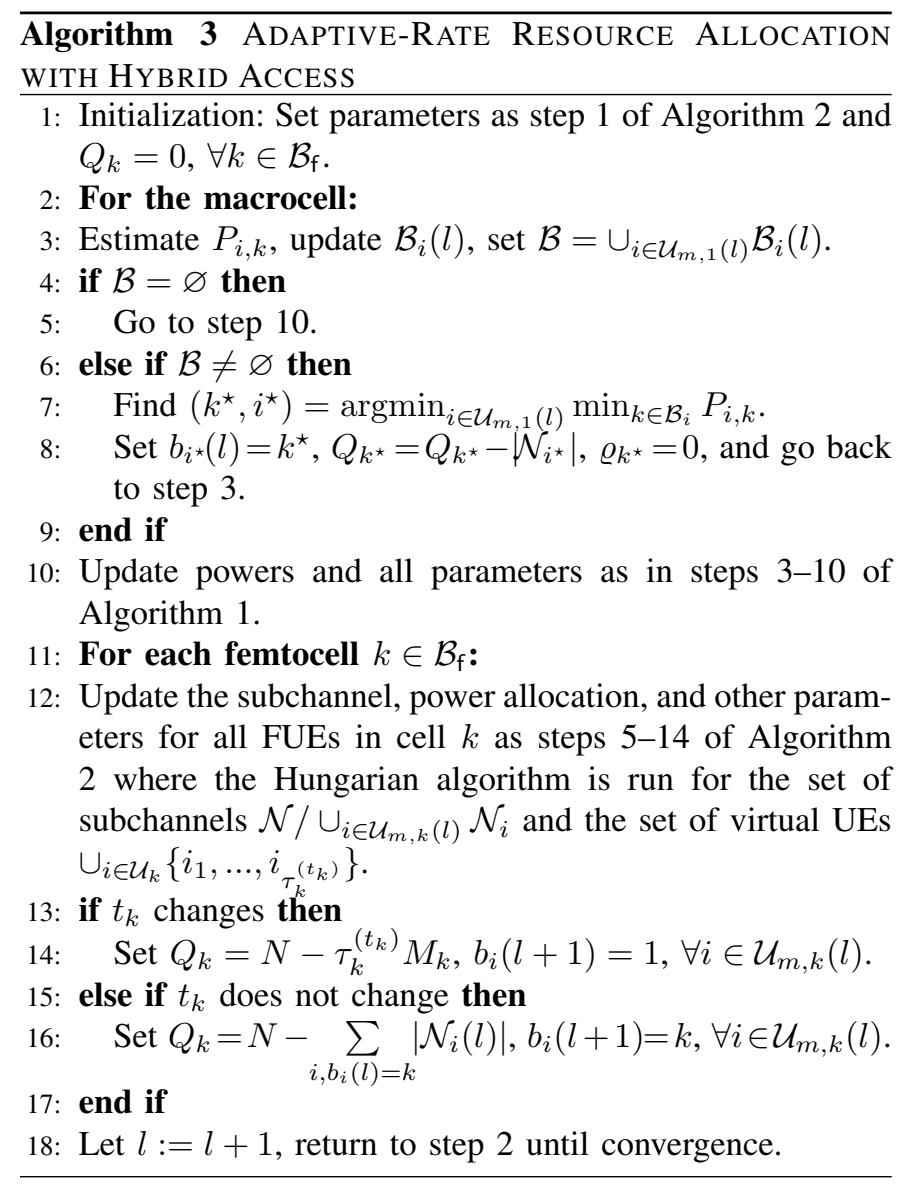

l. In particular, the couple of FBS and MUE requiring the smallest transmission power will be chosen for association in each iteration (step 7). Other operations corresponding to SA, $\mathrm{PA}$, and updates of various parameters are performed as in Algorithm 2.

Algorithm 3 can be implemented as follows. To complete the tasks in steps 3-9, each MUE $i$ upon estimating $P_{i, k}$ for all potential FBSs $k \in \mathcal{B}_{i}$ will send a connection request to the FBS that requires the minimum power (i.e., $\min _{k \in \mathcal{B}_{i}} P_{i, k}$ ) together the value of $P_{i, k}$. If a particular FBS $k$ receives several connection requests then the FBS will grant the connection to only one MUE with the smallest value of $P_{i, k}$. Signaling information needed to exchange the connection request and granting messages can be accommodated by available control channels. In steps 13-14, if the next pair $\left(s_{k}, \tau_{k}\right)$ with index $t_{k}$ in the sorted list $\Theta_{k}$ in femtocell $k$ is chosen (i.e., $t_{k}$ is increased by one) then FBS $k$ simply requests all associated MUEs to change their connections back to the MBS. Otherwise, if $t_{k}$ remains unchanged then FBS $k$ simply updates parameter $Q_{k}$ based on the new SA assignment decisions in step 12.

\section{Numerical Results}

We obtain numerical results for two different networks (with a small and large number of femtocells and UEs) to evaluate the efficacy of our proposed algorithms. The network setting and UE placement for our simulations are illustrated in Fig. 1, where MUEs and FUEs are randomly located inside circles of

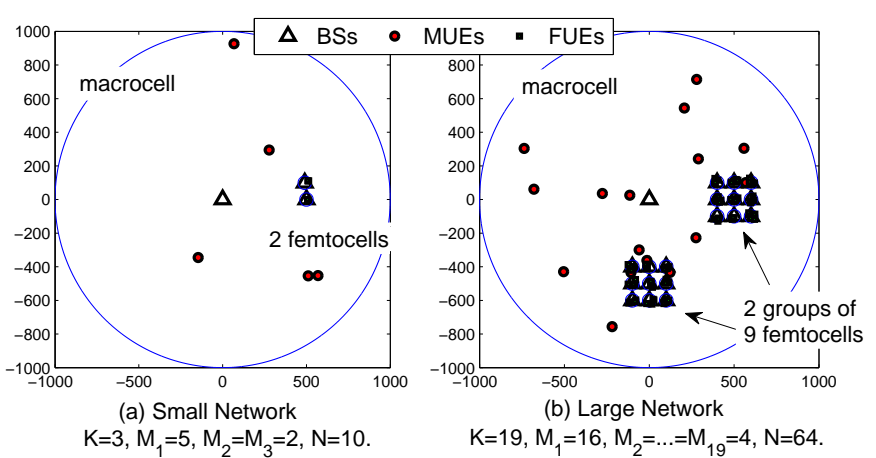

Fig. 1. Macrocell-femtocell networks used in simulation

TABLE III

TARGET SINRS FOR DIFFERENT CONSTELLATION SIZES WITH TARGET BER $\bar{P}_{e}=10^{-3}$.

\begin{tabular}{|c|c|c|c|c|c|}
\hline$s$-QAM & 4 & 16 & 64 & 256 & 1024 \\
\hline bits/symbol & 2 & 4 & 6 & 8 & 10 \\
\hline $\bar{\gamma}(s)$ & 9.55 & 45.11 & 179.85 & 694.17 & 2667.32 \\
\hline
\end{tabular}

radii of $r_{1}=1000 \mathrm{~m}$ and $r_{2}=30 \mathrm{~m}$, respectively. The power channel gains $h_{i j}^{n}$ are generated by considering both Rayleigh fading, which is represented by an exponentially distributed random variable with the mean value of one, and the path loss $L_{i j}=A_{i} \log _{10}\left(d_{i j}\right)+B_{i}+C \log _{10}\left(\frac{f_{c}}{5}\right)+W L \times n_{i j}$, where $d_{i j}$ is the distance from UE $j$ to BS $i ;\left(A_{i}, B_{i}\right)$ are set as $(36,40)$ and $(25,45)$ for MBS and FBSs, respectively; $C=20, f_{c}=2.5 \mathrm{GHz} ; W L$ is the wall-loss parameter, $n_{i j}$ is the number of walls between BS $i$ and UE $j$. This pathloss model is chosen according to the general form of the path loss formula (4.23) in [24], which is suggested by the WINNER II channel modeling project. The noise power is set as $\eta_{i}=10^{-13} W, \forall i \in \mathcal{B}$.

To obtain simulation results, we use two modulation schemes (4- and 16-QAM) for MUEs and five modulation schemes (4-, 16-, 64-, 256- and 1024-QAM) for FUEs. Moreover, we choose the target BER $\bar{P}_{e}=10^{-3}$ whose target SINRs corresponding to different modulation schemes, which can be calculated by using (5), are given in Table III. Each simulation result is obtained by taking the average over 20 different runs where for each run, UEs are randomly located and a feasible SA matrix for MUEs $\mathbf{A}_{1}$ is chosen so that each MUE is assigned $\frac{N}{M_{1}}$ subchannels.

\section{A. Performance of Proposed Algorithms}

In Fig. 2, we show the total minimum spectral efficiency of all femtocells (i.e., the optimal objective value of (13)) versus the constellation size of FUEs $\left(s^{f}\right)$ for the small network due to both optimal and sup-optimal algorithms (Algorithm 1), which are presented in Section III. As can be seen, for the low constellation sizes (i.e., low target SINRs), Algorithm 1 can achieve almost the same spectral efficiency as the optimal one while for higher values of $s^{\mathrm{f}}$, Algorithm 1 results in just slightly lower spectral efficiency than that due to the optimal one. Moreover, when we increase the value of parameter $V$, 


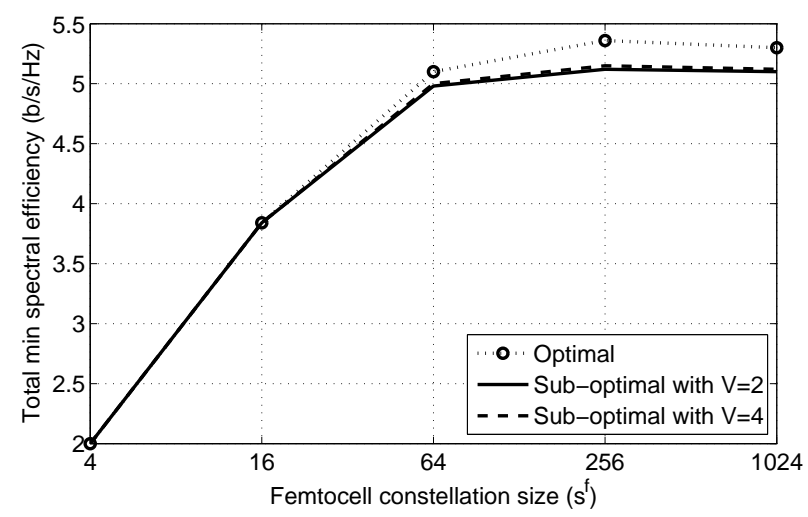

Fig. 2. Total minimum spectral efficiency for small network under optimal and sup-optimal algorithms for $s^{\mathrm{m}}=4, W L=5 \mathrm{~dB}, P_{\mathrm{m}}^{\max }=P_{\mathrm{f}}^{\max }=0.01 \mathrm{~W}$.

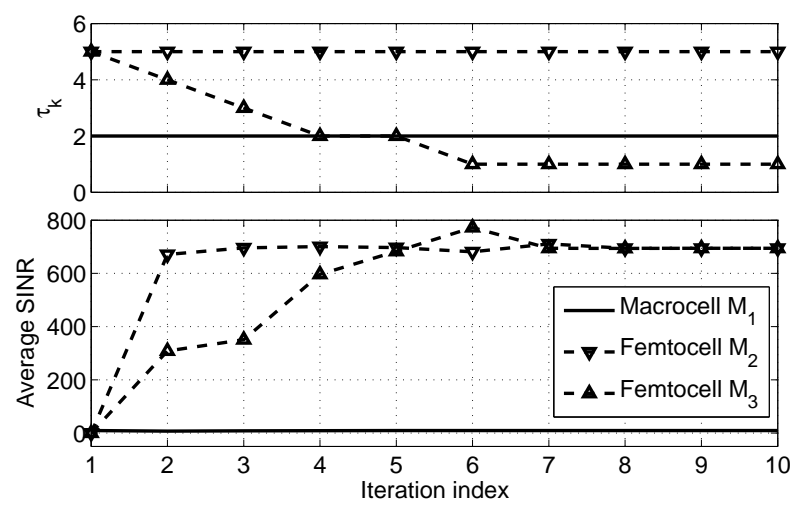

Fig. 3. Minimum number of subchannels assigned for FUEs and average SINRs versus iteration index where $s^{\mathrm{m}}=4, s^{\mathrm{f}}=256, W L=5 \mathrm{~d} B$, $P_{\mathrm{m}}^{\max }=P_{\mathrm{f}}^{\max }=0.01 \mathrm{~W}$.

a slightly better performance can be achieved. We then plot the minimum number of subchannels assigned for FUEs and the average SINRs over assigned subchannels versus iteration index for the small network in Fig. 3. This figure illustrates the convergence of Algorithm 1 in terms of both user SINR and number of assigned subchannels per FUE.

Fig. 4 presents the total femtocell minimum spectral efficiency versus the QAM constellation size, which is obtained by running Algorithm 1 for the large network. This figure shows that the total minimum spectral efficiency increases and then decreases as femtocell constellation size increases; and it decreases as the constellation size of MUEs increases. These results can be interpreted as follows. Higher constellation sizes have higher target SINRs, which require higher transmission power and produce more interference to other users in the network. Therefore, the power constraints of FUEs and MUEs are more likely to be violated for higher constellation sizes (e.g., 254 or 1024), which limits the number of subchannels allocated for each FUE. Moreover, for low modulation levels (therefore, low target SINRs of FUEs) the spectral efficiency on each assigned subchannel increases with the increasing modulation level while the number of subchannels assigned

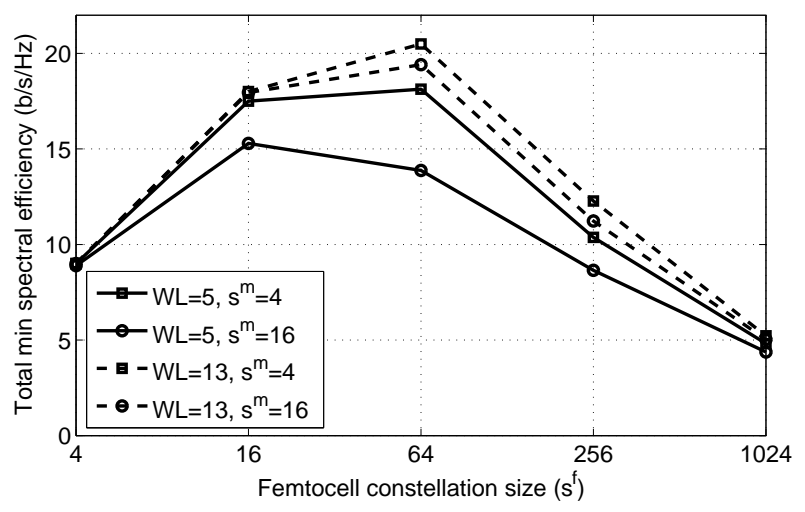

Fig. 4. Total minimum spectral efficiency versus QAM constellation size of femtocells with $P_{\mathrm{m}}^{\max }=P_{\mathrm{f}}^{\max }=0.01 \mathrm{~W}$.

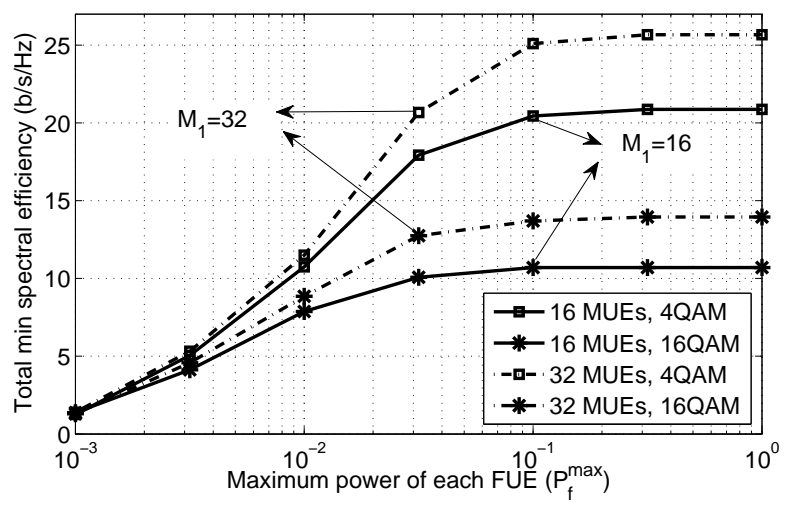

Fig. 5. Total minimum spectral efficiency versus $P_{\mathrm{f}}^{\max }$ for the large network with $s^{\mathrm{f}}=256, W L=5 \mathrm{~dB}, P_{\mathrm{m}}^{\max }=0.01 \mathrm{~W}$.

for each FUE does not decrease too much. Moreover, the total minimum spectral efficiency of femtocells increases with the increasing wall loss value $W_{l}$. This is because the higher wall loss reduces both co-tier and cross-tier interference to users of both tiers.

In Figs. 5 and 6, we plot the total femtocell minimum spectral efficiency versus the maximum power of FUEs $\left(P_{\mathrm{f}}^{\max }\right)$ and MUEs $\left(P_{\mathrm{m}}^{\max }\right)$, respectively for different modulation levels of MUEs (the modulation scheme of FUEs is 256-QAM). These figures show that the total minimum spectral efficiency increases with the increases of maximum power budgets, $P_{f}^{\max }$ or $P_{\mathrm{m}}^{\max }$. However, this value is saturated as the maximum power budgets $P_{\mathrm{f}}^{\max }$ or $P_{\mathrm{m}}^{\max }$ become sufficiently large. In addition, as the number of MUEs increases, the total femtocell minimum spectral efficiency increases thanks to the better diversity gain offered by the macro tier.

In Fig. 7, we show the total minimum spectral efficiency of femtocells versus the QAM constellation sizes for the downlink by running the resource allocation algorithm with the power constraints (24) and SA weights (27). This figure shows that the proposed algorithm works well for the downlink system where these results are similar to those for the uplink case presented in Fig. 4. 


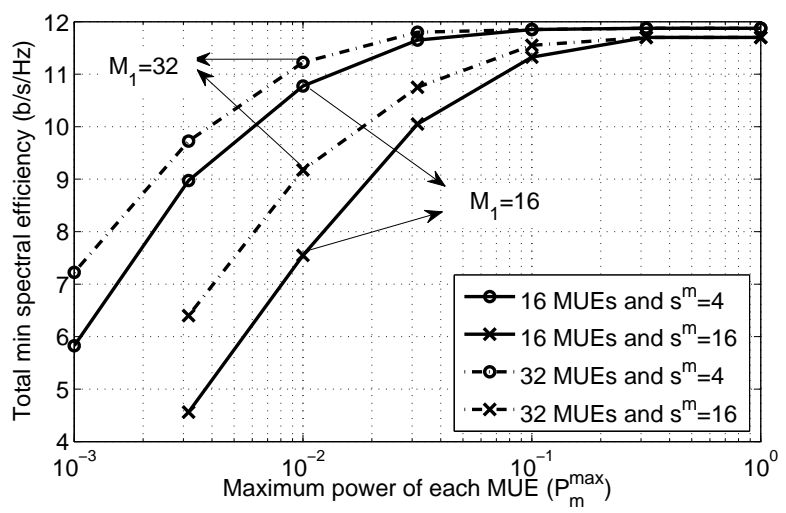

Fig. 6. Total minimum spectral efficiency versus $P_{\mathrm{m}}^{\max }$ for the large network with $s^{\mathrm{f}}=256, W L=5 \mathrm{~dB}, P_{\mathrm{f}}^{\max }=0.01 \mathrm{~W}$.

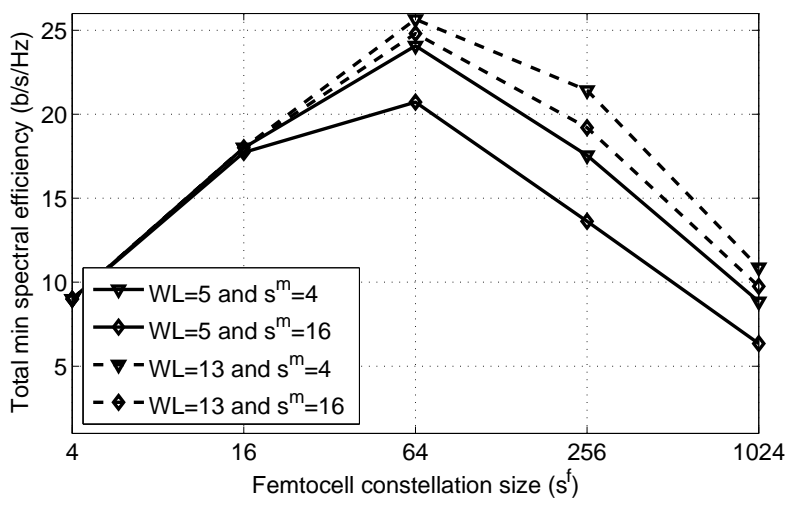

Fig. 7. Total minimum spectral efficiency of femtocells in the downlink with $W L=5 d B, P_{\mathrm{BS}, m}^{\max }=0.2 W, P_{\mathrm{BS}, f}^{\max }=0.05 W$.

Fig. 8 presents the total minimum spectral efficiency of femtocells versus the wall loss parameter $(W L)$ achieved by the fixed rate algorithm (i.e., Algorithm 1) and the adaptiverate algorithm (i.e., Algorithm 2) whose results are indicated by "Max-Fixed Rate" and "Multi-Rate" in this figure, respectively. In Algorithm 2, each femtocell can choose one best modulation scheme among five schemes $\left(s^{f}=\right.$ $4,16,64,256,1024)$ while Algorithm 1 employs the maximum modulation level for all femtocells. This figure demonstrates that the great performance gain can be achieved by exploiting the adaptive rate feature in performing resource allocation for FUEs.

In Fig. 9, we illustrate the total minimum spectral efficiency of all femtocells versus $P_{f}^{\max }$ under the hybrid and closed access strategies, which are obtained by Algorithm 3 and Algorithm 2, respectively. These results correspond to the large nework with 32 MUEs and $W_{l}=5 d B$. As can be seen, the hybrid access strategy achieves higher performance than that under closed access scheme. Moreover, the performance gap between two strategies becomes larger with increasing maximum power budget of FUEs. Under the closed access, the total spectral efficiency of femtocells is indeed limited by MUEs that are located far away from the MBS and close to

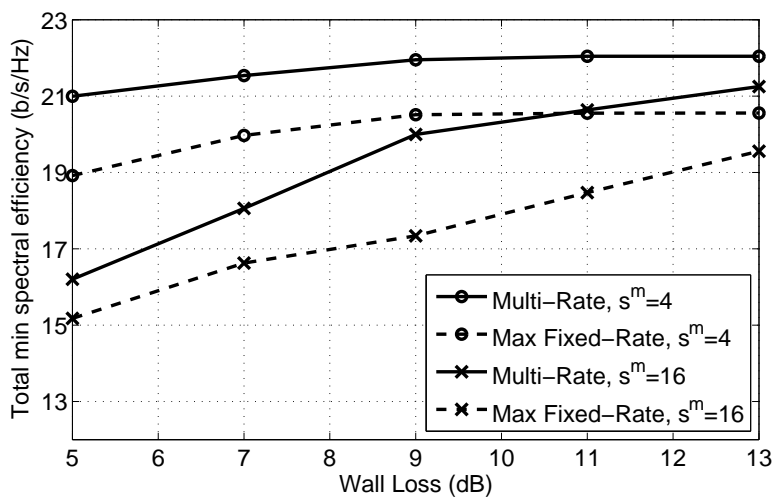

Fig. 8. Total minimum spectral efficiency versus wall loss for Algorithm 2 with $P_{\mathrm{m}}^{\max }=P_{\mathrm{f}}^{\max }=0.01 \mathrm{~W}$.

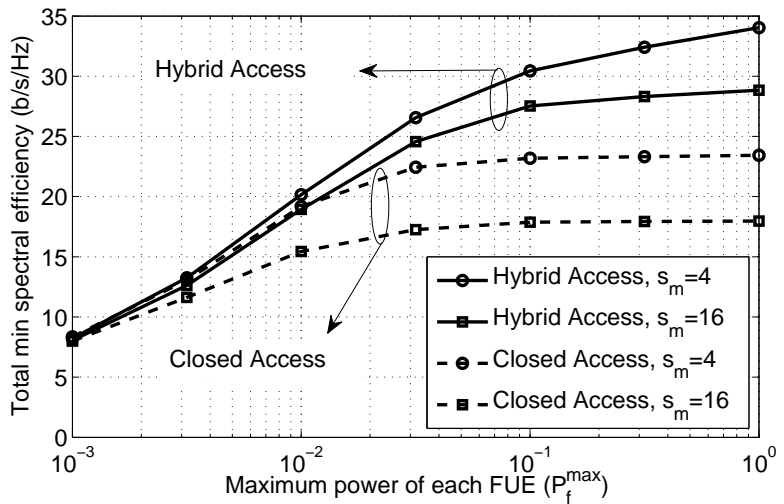

Fig. 9. Total minimum spectral efficiency versus $P_{\mathrm{f}}^{\max }$ under the adaptive rate and hybrid access strategy for $P_{\mathrm{m}}^{\max }=0.01 W, M_{1}=32$ and $W L=5 \mathrm{~dB}$.

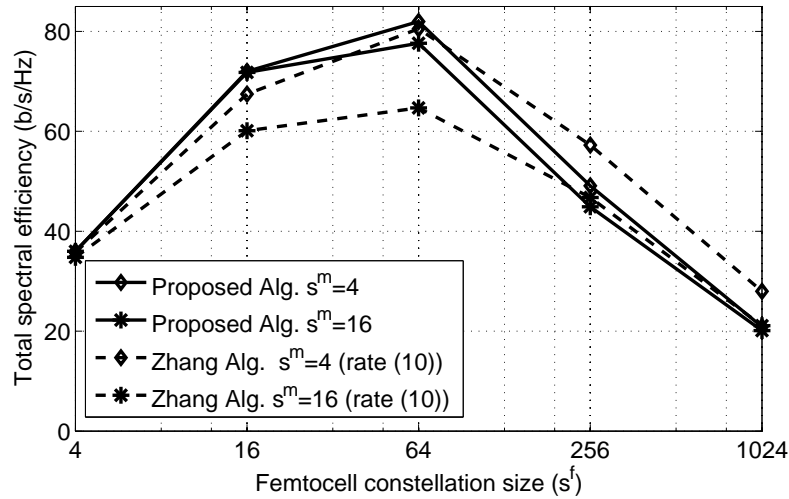

Fig. 10. Total spectral efficiency versus QAM constellation size of FUEs with $P_{\mathrm{m}}^{\max }=P_{\mathrm{f}}^{\max }=0.01 \mathrm{~W}$.

some FBSs. The hybrid access strategy enables these victim MUEs to connect with nearby FBSs which mitigates this problem and increases the spectral efficiency of the femto tier. 


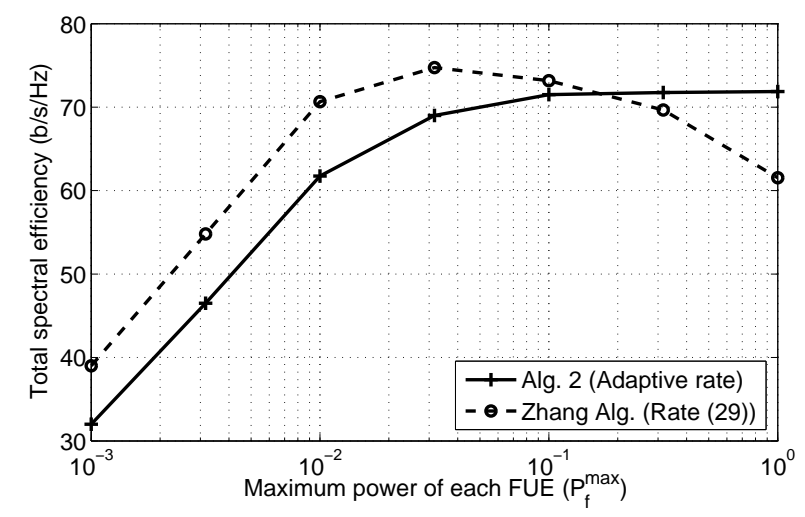

Fig. 11. Total spectral efficiency versus maximum power of each FUE with $P_{\mathrm{m}}^{\max }=0.01 \mathrm{~W}$.

\section{B. Performance Comparison with Zhang's Algorithm [27]}

Performance comparison between our proposed algorithm and that in [27] is presented in the following. To obtain the simulation results in Fig. 10, the spectral efficiency of each FUE is obtained by running Zhang's algorithm and calculating the spectral efficiency by using the fixed-rate formula (10). In Fig. 10, we illustrate the total spectral efficiency of all FUEs in all femtocells due to Zhang's algorithm [27] and Algorithm 1 as we vary the QAM constellation size of each FUE $s^{f}$. This figure shows that for a fixed constellation size employed by MUEs, the total spectral efficiency achieved by Zhang's algorithm is lower than ours for low values of $s^{\mathrm{f}}$ while Zhang's algorithm results in slightly higher spectral efficiency than our algorithm for larger values of $s^{\mathrm{f}}$. The slightly better performance of Zhang's algorithm comes at the cost of severely unfair resource sharing among FUEs as we will present in Fig. 12. It is worth to recall that Zhang et. al. aim to maximize the total throughput without considering user fairness while our formulation focuses on achieving an equal rate for FUEs in each femtocell (i.e., max-min fairness).

Fig. 11 illustrates the total spectral efficiency of Zhang's algorithm and our proposed algorithms with rate adaptation (i. e., Algorithm 2) as we vary the maximum power of each FUE $P_{f}^{\max }$. Here, the spectral efficiency of FUEs under Zhang's algorithm is calculated by using the adaptive rate formula (29). As evident, the total spectral efficiency due to Zhang's algorithm is higher than ours for low values of $P_{\mathrm{f}}^{\max }$ but Zhang's algorithm performs worse compared to our algorithm if $P_{\mathrm{f}}^{\max }$ becomes sufficiently large. The superior performance of Zhang's algorithm in the low-power regime is again due to its unfair resource sharing nature. In fact, Zhang's algorithm does not account for the co-tier interference among femtocells, which explains why it performs worse compared to our algorithm in the high-power regime. This is because the transmission powers of FUEs increase with the larger power budget, which results in higher femtocell co-tier interference. The high co-tier interference degrades the performance of Zhang's algorithm since this type of interference is not managed in their work.

To compare the fairness of Zhang's and our proposed

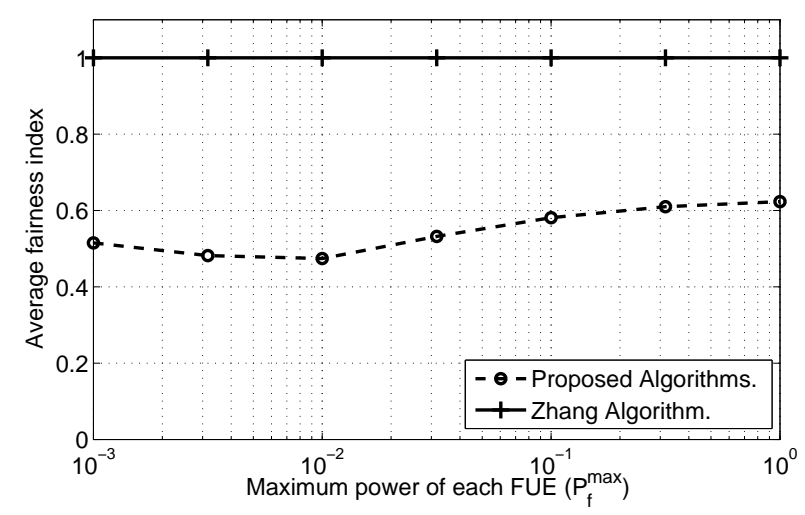

Fig. 12. Fairness index versus maximum power of each FUE with $P_{\mathrm{m}}^{\max }=$ $0.01 W$.

algorithms, we present the average fairness index achieved by FUEs in each femtocell, which is calculated as [26]

$$
F I_{k}=\left(\sum_{i=1}^{M_{k}} R_{i}\right)^{2} / M_{k}\left(\sum_{i=1}^{M_{k}} R_{i}^{2}\right) .
$$

This fairness index is widely employed in the literature to evaluate the level of fairness achieved by resource allocation algorithms where an algorithm is fairer if its fairness index is higher and close to the maximum value of one and vice versa. Fig. 12 shows the average fairness index achieved by Zhang's and our proposed algorithms. As evident, our algorithm provides maximum fairness for FUEs in each femtocell (since the rates of FUEs in each femtocell are equal), while the average fairness index of Zhang's algorithm is around 0.50.6 , which is quite low. This implies that Zhang's algorithm may result in big differences in the rates achieved FUEs in each femtocell, which is not very desirable. Considering that the total spectral efficiency due to Zhang's and our proposed algorithms is not much different while Zhang's algorithm is highly unfair, we would conclude that our proposed algorithms better balance between the throughput and fairness compared to Zhang's algorithm.

\section{CONCLUSION}

We have proposed centralized optimal and distributed resource allocation algorithms that maximizes the total minimum spectral efficiency of the femtocell network while ensuring fairness among FUEs and QoS protection for all MUEs. Moreover, the proposed algorithm has been extended for three scenarios: downlink context; adaptive-rate systems where FUEs in each femtocell can adaptively choose one in a predetermined set of modulation schemes; and hybrid access design where MUEs can associate with nearby FBSs to improve the performance of the femto tier. Extensive numerical results have been presented to demonstrate the impacts of different system parameters on the network performance and the efficacy of our proposed resource allocation algorithms. 


\section{REFERENCES}

[1] J. G. Andrews, H. Claussen, M. Dohler, S. Rangan, and M. C. Reed, "Femtocells: Past, present, and future," IEEE J. Sel. Areas Commun., vol. 30, no. 3, pp. 497-508, Apr. 2012.

[2] D. Lopez-Perez, A. Valcarce, G. de la Roche, and J. Zhang , "OFDMA femtocells: A roadmap on interference avoidance," IEEE Commun. Mag., vol. 47, no.9, pp. 41-48, Sep. 2009.

[3] G. Li and H. Liu, "Downlink radio resource allocation for multi-cell OFDMA system,” IEEE Trans. Wireless Commun., vol. 5, no. 12, pp. 3451-3459, Dec. 2006.

[4] N. Saquib, E. Hossain, L. B. Le, and D. I. Kim, "Interference management in OFDMA femtocell networks: Issues and approaches," IEEE Commun. Mag., vol. 19, no. 3, pp. 86-95, Jun. 2012.

[5] C.-H. Ko. and H.-Y, Wei, "On-demand resource-sharing mechanism design in two-tier OFDMA femtocell networks," IEEE Trans. Veh. Technol., vol. 60, no. 3, pp. 1059-1071, Mar. 2011.

[6] Y. Sun, R.P. Jover, and X. Wang, "Uplink interference mitigation for OFDMA femtocell networks," IEEE Trans. Wireless Commun., vol. 11, no. 2, pp. 614-625, Feb. 2012.

[7] Y.-S. Liang, W.-H. Chung, G.-K. Ni, I.-Y. Chen, H. Zhang, and S.Y. Kuo, "Resource allocation with interference avoidance in OFDMA femtocell networks," IEEE Trans. Veh. Technol., vol. 61, no. 5, pp. 2243 2255, Jun. 2012.

[8] W. C. Cheung, T. Q. S. Quek, and M. Kountouris, "Throughput optimization, spectrum allocation, and access control in two-tier femtocell networks," IEEE J. Sel. Areas Commun., vol. 30, no. 3, pp. 561-574, Apr. 2012.

[9] L. B. Le, D. Niyato, E. Hossain, D. I. Kim, and D. T. Hoang, "QoS-aware and energy-efficient resource management in OFDMA femtocells," IEEE Trans. Wireless Commun., vol. 12, no. 1, pp. 180-194, Jan. 2013.

[10] J.-H. Yun and K. G. Shin, "Adaptive interference management of OFDMA femtocells for co-channel deployment," IEEE J. Sel. Areas Commun., vol. 29, no. 6, pp. 1225-1241, Jun. 2011.

[11] R. Xie, F. R. Yu, and H. Ji, "Energy-efficient spectrum sharing and power allocation in cognitive radio femtocell networks," in Proc. IEEE INFOCOM, pp. 1665-1673, Mar. 2012.

[12] Z. Lu, T. Bansal, and P. Sinha, "Achieving user-level fairness in openaccess femtocell based architecture," IEEE Trans. Mobile Com., vol. 12, no. 10 , pp. 1943-1954, Oct. 2013.

[13] J. G. Proakis, Digital communications, 2nd ed. McGraw-Hill, 1989.

[14] A. Horn and A. Johnson, Matrix Analysis, 1st ed. Cambridge University Press, 1985.

[15] N. Bambos, S. C. Chen and G. J. Pottie, "Channel access algorithm with active link protection for wireless communication networks with power control", IEEE/ACM Trans. Netw., vol. 8, no. 5, pp. 583-597, Oct. 2000.

[16] G. J. Foschini and Z. Miljanic, "A simple distributed autonomous power control algorithm and its convergence," IEEE Trans. Veh. Technol., vol. 42, no. 4, pp. 641-646, Nov. 1993.

[17] N. Bambos, S. C. Chen, and G. J. Pottie, "Channel access algorithms with active link protection for wireless communication networks with power control," IEEE/ACM Trans. Netw., vol. 8, no. 5, pp. 583-597, Oct. 2000.

[18] V. Y. Pan and Z. Q. Chen, "The complexity of the matrix eigenproblem," in Proc. ACM symposium on Theory of computing, Atlanta, Georgia, United States, 1999.

[19] A. Bojanczyk, "Complexity of solving linear systems in different models of computation," SIAM J. Numerical Analysis, vol. 21, pp. 591-603, 1984.

[20] D. Jungnickel, chapter “Weighted matchings,” in Graphs, Networks and Algorithms, Springer Berlin Heidelberg, vol. 5, pp. 419-456, 2008.

[21] J. Lee and S. Leyffer eds., chapter "Mixed integer nonlinear programming," in The IMA Volumes in Mathematics and its Applications, vol. 154, Springer, 2012.
[22] E. Seneta, Non-Negative Matrices. London, U.K.: G. Allen, 1973.

[23] F. R. Gantmacher, The Theory of Matrices. New York, NY: Chelsea, 1971.

[24] P. Kyosti, J. Meinila, L. Hentila, X. Zhao, T. Jamsa, C. Schneider, M. Narandzic, M. Milojevic, A. Hong, J. Ylitalo, V.-M. Holappa, M. Alatossava, R. Bultitude, Y. de Jong, and T. Rautiainen, WINNER II Channel Models: Eur. Comm., Deliverable IST-WINNER D1.1.2 ver 1.2, Sep. 2007. [Online]. Available: http://projects.celticinitiative.org/winner+/WINNER2-Deliverables/

[25] E. Karipidis, N. D. Sidiropoulos, and Z.-Q. Luo, "Quality of service and max-min fair transmit beamforming to multiple cochannel multicast groups," IEEE Trans. Signal Process., vol. 56, no. 3, pp. 1268-1279, Mar. 2008.

[26] R. Jain, A. Durresi, and G. Babic, "Throughput fairness index: An explanation," ATM Forum Document Number: ATM Forum/990045, Feb. 1999.

[27] H. Zhang, W. Zheng, X. Chu, X. Wen, M. Tao, A. Nallanathan, and D. Lopez-Perez, "Joint subchannel and power allocation in interferencelimited OFDMA femtocells with heterogeneous QoS guarantee," in Proc. IEEE GLOBECOM'2012, pp. 4572-4577, 2012.

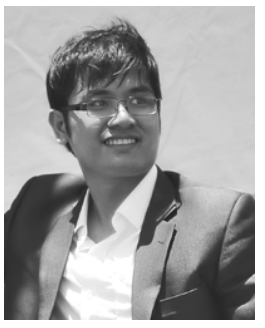

Vu Nguyen Ha (S'11) received the B.Eng. degree from the French training program for excellent engineers in Vietnam (PFIEV), Ho Chi Minh City University of Technology (HCMUT), Vietnam and an addendum degree from de École Nationale Supérieure des Télécommunications de BretagneGroupe des École des Télécommunications, Bretagne, France in 2007. He is currently working toward the Ph.D. degree at the Institut National de la Recherche Scientifique-Énergie, Matériaux et Télécommunications (INRS-EMT), Université du Québec, Montréal, Québec, Canada.

From 2008 to 2011, he was a Research Assistant in the School of Electrical Engineering, University of Ulsan, South Korea. His research interest includes radio resource management for wireless networks with special emphasis on heterogeneous networks.

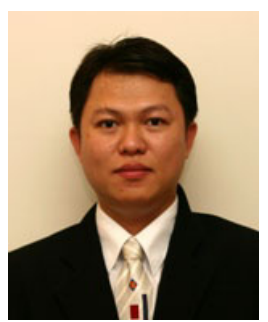

Long Bao Le (S'04-M'07-SM'12) received the B.Eng. (with Highest Distinction) degree from Ho Chi Minh City University of Technology, Vietnam, in 1999, the M.Eng. degree from Asian Institute of Technology, Pathumthani, Thailand, in 2002, and the Ph.D. degree from the University of Manitoba, Winnipeg, MB, Canada, in 2007.

From 2008 to 2010 , he was a postdoctoral research associate with Massachusetts Institute of Technology, Cambridge, MA. Since 2010, he has been an assistant professor with the Institut National de la Recherche Scientifique (INRS), Université du Québec, Montréal, QC, Canada, where he leads a research group working on cognitive radio and dynamic spectrum sharing, radio resource management, network control and optimization. 
TABLE II

SUMMARY OF KEY NOTATIONS

\begin{tabular}{|c|c|}
\hline Notations & Description \\
\hline$\overline{a_{i}^{n}}$ & Subchannel assignment variable for subchannel $n$ and UE $i$ \\
\hline A & Subchannel assignment matrix for all $M$ UEs over $N$ subchannels \\
\hline $\mathbf{A}_{k}$ & Subchannel assignment matrix for UEs in cell $k$ over $N$ subchannels \\
\hline$A_{i}, B_{i}, C$ & Coefficients in path loss formula \\
\hline$b_{i}$ & Base station serving UE $i$ \\
\hline$B W$ & Total bandwidth of all $N$ subchannels \\
\hline $\mathcal{B}$ & Set of all base stations \\
\hline $\mathcal{B}_{\mathrm{f}}$ & Set of all FBSs \\
\hline $\mathcal{B}_{i}$ & Set of potential FBSs for MUE $i$ \\
\hline$c^{n}=\left|\mathcal{U}^{n}\right|$ & Number of elements in set $\mathcal{U}^{n}$ \\
\hline$d_{i j}$ & Distance from UE $j$ to BS $i$ \\
\hline$f_{c}$ & Frequency \\
\hline$f(\gamma(s))$ & Bit error rate (BER) function for SINR $\gamma(s)$ as the constellation size $s$ is adopted \\
\hline$h_{i j}^{n}$ & power channel gain from user $j$ to BS $i$ over subchannel $n$ \\
\hline$I_{i, k}^{n}$ & Effective interference corresponding to MUE $i$ for its connection with BS $k$ on subchannel $n$ \\
\hline$I_{i}^{n}(\mathbf{A}, \mathbf{P})$ & Effective interference corresponding to UE $i$ on subchannel $n$ \\
\hline$K$ & Number of cells \\
\hline$L_{i j}$ & Path loss \\
\hline$M_{k}$ & Number of UEs served by base station $k$ \\
\hline$M_{\mathrm{m}}, M_{\mathrm{f}}$ & Numbers of MUEs and FUEs, respectively \\
\hline $\mathfrak{M}$ & Set of constellation sizes \\
\hline $\mathcal{N}$ & Set of all subchannels \\
\hline $\mathcal{N}_{i}$ & Set of subchannels assigned to UE $i$ \\
\hline$N$ & Number of subchannels \\
\hline$n_{i j}$ & Number of walls between BS $i$ and UE $j$ \\
\hline$p_{i}^{n, \min }$ & Required transmission power of UE $i$ over its assigned subchannel $n$ \\
\hline$p_{i}^{n}$ & Transmission power of UE $i$ over subchannel $n$ \\
\hline$P_{i, k}$ & Estimated required power for MUE $i$ connecting with BS $k$ \\
\hline$P_{i}^{\max }$ & Maximum transmission power of UE $i$ \\
\hline$P_{\mathrm{BS}, k}^{\mathrm{max}}$ & Maximum power of BS $k$ in the downlink \\
\hline $\mathbf{P}$ & Power allocation matrix whose elements are $p_{i}^{n}$ \\
\hline $\mathbf{P}_{k}$ & Power allocation matrix for UEs over $N$ subchannels in cell $k$ \\
\hline $\bar{P}_{e}$ & Target BER \\
\hline$Q_{k}$ & Number of unused subchannels at FBS $k$ \\
\hline $\mathbb{Q}()$. & Q function \\
\hline$r_{i}^{n}(\mathbf{A}, \mathbf{P})$ & Spectral efficiency (bits/s/Hz) achieved by FUE $i$ on subchannel $n$ \\
\hline$R_{i}(\mathbf{A}, \mathbf{P})$ & Spectral efficiency (bits/s/Hz) achieved by FUE $i$ \\
\hline$s$ & Constellation size corresponding to the $s$-QAM modulation scheme \\
\hline$s^{k}$ & Constellation size of the MQAM modulation utilized in cell $k$ \\
\hline$s_{\max }$ & Maximum allowable constellation size in the set $\mathfrak{M}$ \\
\hline$s^{\mathrm{m}}, s^{\mathrm{f}}$ & Constellation size of the MQAM modulation employed in macrocell and femtocells, respectively \\
\hline $\mathcal{U}_{k}$ & Set of all UEs served by base station $k$ \\
\hline $\mathcal{U}_{m, k}$ & Set of MUEs connecting with BS $k$ \\
\hline $\mathcal{U}, \mathcal{U}_{\mathrm{m}}, \mathcal{U}_{\mathrm{f}}$ & Set of all UEs, MUEs and FUEs, respectively \\
\hline $\mathcal{U}^{n}$ & Set of UEs in both tiers that are assigned subchannel $n$ \\
\hline$w_{i}^{n}$ & Assignment weight of UE $i$ over subchannel $n$ \\
\hline$W_{k}^{2}$ & Total minimum weight of subchannel assignments in cell $k$ \\
\hline$W L$ & Wall-loss parameter \\
\hline$\alpha_{i}^{n}, \theta_{i}^{n}, \delta_{i}^{n}, \delta_{i}^{n}$ & Other factors for calculating scaling factor $\chi_{i}^{n}$ \\
\hline $\bar{\gamma}(s)$ & Target SINR corresponding to constellation size $s$ \\
\hline & Target SINR of UE $i$ over subchannel $n$ \\
\hline$\Gamma_{i}^{n}(\mathbf{A}, \mathbf{P})$ & SINR of UE $i$ over subchannel $n$ \\
\hline$\eta_{i}^{n}$ & Noise power at $\mathrm{BS} i$ on subchannel $n$ \\
\hline & Number of subchannels assigned for each UE in cell $k$ \\
\hline$\chi_{i}^{n}$ & Scaling factor in SA weight \\
\hline$\Omega\{\mathbf{A}\}$ & List of all potential SA solutions \\
\hline$\Omega^{*}\{\mathbf{A}\}$ & Sorted list of $\Omega\{\mathbf{A}\}$ in decreasing order of $\sum_{k=2}^{K} \tau_{k}$ \\
\hline
\end{tabular}

